\title{
False-Name Manipulation in Weighted Voting Games is Hard for Probabilistic Polynomial Time
}

\author{
Anja Rey \\ Jörg Rothe \\ Institut für Informatik \\ Heinrich-Heine-Universität Düsseldorf \\ 40225 Düsseldorf \\ Germany
}

REY@CS.UNI-DUESSELDORF.DE

\begin{abstract}
False-name manipulation refers to the question of whether a player in a weighted voting game can increase her power by splitting into several players and distributing her weight among these false identities. Relatedly, the beneficial merging problem asks whether a coalition of players can increase their power in a weighted voting game by merging their weights. For the problems of whether merging or splitting players in weighted voting games is beneficial in terms of the Shapley-Shubik and the normalized Banzhaf index, merely NP-hardness lower bounds are known, leaving the question about their exact complexity open. For the Shapley-Shubik and the probabilistic Banzhaf index, we raise these lower bounds to hardness for PP, "probabilistic polynomial time," a class considered to be by far a larger class than NP. For both power indices, we provide matching upper bounds for beneficial merging and, whenever the new players' weights are given, also for beneficial splitting, thus resolving previous conjectures in the affirmative. Relatedly, we consider the beneficial annexation problem, asking whether a single player can increase her power by taking over other players' weights. It is known that annexation is never disadvantageous for the Shapley-Shubik index, and that beneficial annexation is NP-hard for the normalized Banzhaf index. We show that annexation is never disadvantageous for the probabilistic Banzhaf index either, and for both the Shapley-Shubik index and the probabilistic Banzhaf index we show that it is NP-complete to decide whether annexing another player is advantageous. Moreover, we propose a general framework for merging and splitting that can be applied to different classes and representations of games.
\end{abstract}

\section{Introduction}

Algorithmic game theory has been studied intensely in recent years (Nisan, Roughgarden, Tardos, \& Vazirani, 2007; Chalkiadakis, Elkind, \& Wooldridge, 2011). This paper focuses on the algorithmic and complexity-theoretic aspects of problems from cooperative game theory. The central question studied is whether merging or splitting players in a coalitional game (with transferable utilities) can raise their power in the game. Power indices measure how influential a player is for forming winning coalitions in simple games. The Shapley-Shubik and the Banzhaf power indices are most prominent among such measures (Shapley \& Shubik, 1954; Banzhaf III, 1965). Roughly speaking, in simple games they indicate, respectively, in how many "orders of support" and with what probability a player can swing the outcome of a coalition by joining or leaving it.

Weighted voting games are an important class of succinctly representable, simple games. They can be used to model cooperation among players in scenarios where each player is assigned a weight, and a coalition of players wins if and only if their joint weight meets or exceeds a given 
quota. Typical real-world applications of weighted voting games include decision-making in legislative bodies (e.g., parliamentary voting) and shareholder voting (for further concrete applications and literature pointers see Chalkiadakis et al., 2011). In particular, the algorithmic and complexity-theoretic properties of problems related to weighted voting have been studied in depth (for an overview see, e.g., Elkind, Chalkiadakis, \& Jennings, 2008; Elkind, Goldberg, Goldberg, \& Wooldridge, 2009; Bachrach, Elkind, Meir, Pasechnik, Zuckerman, Rothe, \& Rosenschein, 2009; Zuckerman, Faliszewski, Bachrach, \& Elkind, 2012; Elkind, Pasechnik, \& Zick, 2013; Chalkiadakis et al., 2011).

For weighted voting games, Bachrach and Elkind (2008) were the first to study the complexity of false-name manipulation: Is it possible for a player to increase her power by splitting into several players and distributing her weight among them? Such a player may have an incentive to manipulate the game via introducing false names. Relatedly, they also ask whether merging their weights can help any two players in a weighted voting game to increase their power.

\begin{tabular}{|c|c|c|}
\hline PI-BENEFICIALMERGE & PI-BENEFICIALSPLIT & PI-ANNEXATION \\
\hline - open question * & $\begin{array}{l}\text { - NP-hard (Shapley-Shubik } \\
\text { index, } m=2)^{*} \ddagger\end{array}$ & $\begin{array}{l}\text { - never disadv. (Shapley- } \\
\text { Shubik index })^{\# \ddagger}\end{array}$ \\
\hline $\begin{array}{l}\text { - NP-hard (Shapley-Shubik } \\
\text { index })^{\dagger+}\end{array}$ & & $\begin{array}{c}\text { - never disadv. (probabilis- } \\
\text { tic Banzhaf index) }\end{array}$ \\
\hline $\begin{array}{l}\text { - in PP (Shapley-Shubik in- } \\
\quad \text { dex, }\|S\|=2)^{\S}\end{array}$ & & - NP-complete $(\|S\|=1)^{\text {II }}$ \\
\hline $\begin{array}{l}\text { - in } \mathrm{P} \text { (probabilistic Banzhaf } \\
\quad \text { index, }\|S\|=2)^{\mathbb{I}}\end{array}$ & $\begin{array}{l}\text { - in } \mathrm{P}(\text { probabilistic Banzhaf } \\
\text { index, } m=2)^{\mathrm{q}}\end{array}$ & \\
\hline - PP-complete ${ }^{\mathbb{I}}$ & - PP-hard ${ }^{\mathbb{I}}$ & - NP-hard ${ }^{\mathbb{I I}}$ \\
\hline $\begin{array}{l}* \text { (Bachrach \& Elkind, 2008) } \\
\dagger \text { (Aziz \& Paterson, 2009) } \\
* \text { (Aziz, Bachrach, Elkind, \& P } \\
\S \text { (Faliszewski \& Hemaspaandr } \\
\text { II this paper } \\
{ }^{\#} \text { (Felsenthal \& Machover, } 199\end{array}$ & $\begin{array}{l}\text { aterson, 2011) } \\
\text { a, 2009) }\end{array}$ & \\
\hline
\end{tabular}

Table 1: Overview of the history of complexity results of beneficial merging, splitting, and annexation for the probabilistic Banzhaf index and the Shapley-Shubik index. Key: $\|S\|$ denotes the size of a merging coalition and $m$ is the number of players a given player splits into.

Table 1 gives an overview of the development of these problems' complexity results for the power indices studied here, the probabilistic Banzhaf index and the Shapley-Shubik index, and we briefly elaborate on them now. Merging and extending the results of Bachrach and Elkind (2008) and Aziz and Paterson (2009), Aziz et al. (2011) in particular study the problem of whether 
merging or splitting players in weighted voting games is beneficial in terms of the Shapley-Shubik index (Shapley, 1953; Shapley \& Shubik, 1954) and the normalized Banzhaf index (Banzhaf III, 1965) (see Section 2 for formal definitions). These results, however, provide merely NP-hardness lower bounds. Aziz et al. (2011, p. 72, Remark 13) note that "it is quite possible that our problems are not in NP" (and thus are not NP-complete). Faliszewski and Hemaspaandra (2009) provide the best known upper bound for the beneficial merging problem for two players with respect to the Shapley-Shubik index: It is contained in the class PP, "probabilistic polynomial time," which is considered to be by far a larger class than NP, and they conjecture that this problem is PP-complete. We observe that the same arguments give a PP upper bound for beneficial merging also in terms of the probabilistic Banzhaf index. ${ }^{1}$ In contrast to the normalized Banzhaf index and the ShapleyShubik index, we show for the probabilistic Banzhaf index that the problems of raising power by merging or splitting are in $\mathrm{P}$ for coalitions of size two and a split into two players, respectively.

Furthermore, we bridge the gap between the NP-hardness lower bound and the PP upper bound by proving that beneficial merging and splitting (if the new weights are given in the problem instance) are PP-complete problems both for the Shapley-Shubik and the probabilistic Banzhaf index. Beneficial splitting in general (i.e., if the number of new false identities is given, but not their actual weights) is PP-hard for the same two indices. Thus, none of these six problems can be in NP, unless the polynomial hierarchy collapses to its first level, which is considered highly unlikely.

Felsenthal and Machover (1995) distinguish two types of merging, voluntarily and involuntarily, and show for the latter, a bloc of two players (a.k.a. annexation of one player by another), that it is never disadvantageous for the Shapley-Shubik index, but can be disadvantageous for the normalized Banzhaf index. For the latter index, Aziz et al. (2011) show that it is NP-hard to decide whether a player can benefit from annexing other players. We show that it is NP-complete to decide whether annexing another player is advantageous for the Shapley-Shubik index, as well as for the probabilistic Banzhaf index. We also show that annexation can never be disadvantageous for the probabilistic Banzhaf index, which thus behaves like the Shapley-Shubik index in this regard.

A further contribution of our paper is that we propose a general framework for merging and splitting that can be applied to various classes or representations of games. Introducing new properties of merging and splitting functions, consistency and independence, which in particular are satisfied by the standard merging and splitting functions for weighted voting games, we can generalize the $\mathrm{P}$ results for the probabilistic Banzhaf index. On the one hand, these properties are desirable for the design of a merging or splitting function; on the other hand, they are an approach for an axiomatic evaluation of such functions. The analysis of further properties are an interesting task for future work. As an example of applying this more general framework to a concrete class of games, we consider threshold network flow games on hypergraphs, a model adapted here from the threshold network flow games introduced by Bachrach and Rosenschein (2009). In unanimity games and with respect to the probabilistic Banzhaf index, we show that splitting is always disadvantageous or neutral, whereas merging is neutral for size-two coalitions, yet advantageous for coalitions with at least three players. This strongly contrasts with the results by Aziz et al. (2011) showing that merging is always disadvantageous and splitting is always advantageous for the normalized Banzhaf index in unanimity weighted voting games. These are only two examples of how different properties of a game or restrictions caused by a certain representation can lead to different behavior when consid-

1. Note that the same arguments cannot be transferred immediately to the corresponding problem for the normalized Banzhaf index. 
ering merging and splitting (for an overview of different classes and representations of games see, for example, Shoham \& Leyton-Brown, 2009).

\section{Preliminaries}

We start by providing the needed concepts and notation from cooperative game theory and complexity theory.

\subsection{Basic Notions from Cooperative Game Theory}

We will need the following concepts from cooperative game theory, see, e.g., the textbooks by Chalkiadakis et al. (2011) and Peleg and Sudhölter (2003).

A coalitional game with transferable utilities, $\mathscr{G}=(N, v)$, consists of a set $N=\{1, \ldots, n\}$ of players (or, synonymously, agents) and a coalitional function $v: \mathfrak{P}(N) \rightarrow \mathbb{R}$ with $v(\emptyset)=0$, where $\mathfrak{P}(N)$ denotes the power set of $N$. We consider different classes of games with certain properties. For example, a coalitional game is monotonic if $v(B) \leq v(C)$ whenever $B \subseteq C$ for coalitions $B, C \subseteq N$, and it is simple if it is monotonic and $v: \mathfrak{P}(N) \rightarrow\{0,1\}$ maps each coalition $C \subseteq N$ to a value that indicates whether $C$ is successful (i.e., $C$ wins: $v(C)=1$ ) or not (i.e., $C$ loses: $v(C)=0$ ), where we require that the grand coalition $N$ is always winning (i.e., $v(N)=1$ ).

Since the number of coalitions is exponential in the number of players, specifying coalitional games by listing all values of their coalitional function would require exponential space. For algorithmic purposes, however, it is important that these games can be represented succinctly. Weighted voting games (a.k.a. weighted threshold games) are an important class of simple games that are compactly representable (Bilbao, Fernández, Jiménez, \& López, 2002). In such games, each player has a given weight, and a coalition of players is successful if and only if the sum of their weights reaches or exceeds a given threshold, called the quota. Formally, a weighted voting game (WVG) $\mathscr{G}=\left(w_{1}, \ldots, w_{n} ; q\right)$ consists of a quota $q \in \mathbb{N}$ and nonnegative integer ${ }^{2}$ weights $w_{i}, 1 \leq i \leq n$, where $w_{i}$ is the $i$ th player's weight. For each coalition $C \subseteq N$, letting $w(C)$ denote $\sum_{i \in C} w_{i}, C$ wins if $w(C) \geq q$, and it loses otherwise. Requiring the quota to satisfy $0<q \leq w(N)$ ensures that the empty coalition loses and the grand coalition wins. Weighted voting games have been intensely studied from a computational complexity point of view, see, e.g., the work of Elkind et al. (2009) and the book by Chalkiadakis et al. (2011, ch. 4) for an overview.

A weighted majority game $(W M G)$ is the special case of a WVG where the quota is set to $q=\lfloor w(N) / 2\rfloor+1$. WVGs are compactly representable, although not fully expressive (for a simple game that cannot be represented as a WVG see, e.g., Chalkiadakis et al., 2011, Example 4.17). More precisely, every simple game can be represented as a $k$-weighted voting game, which is the intersection of $k$ weighted voting games; the smallest such $k$ is the dimension of the simple game; and weighted voting games are fully expressive only for the class of one-dimensional simple games (again, see the book by Chalkiadakis et al. (2011) for more details).

For a coalitional game $\mathscr{G}=(N, v)$, let $d \mathscr{G}(C, i)=v(C \cup\{i\})-v(C)$ be the marginal contribution of a player $i \in N$ to a coalition $C \subseteq N \backslash\{i\}$. In a simple game, a player $i$ is called pivotal (or "crucial" or "critical") for a coalition $C \subseteq N \backslash\{i\}$ if $C \cup\{i\}$ is successful, but $C$ is not. We have $d_{\mathscr{G}}(C, i)=1$ if player $i$ is pivotal for $C$, and $d_{\mathscr{G}}(C, i)=0$ otherwise. This term can be generalized to nonsimple games, referring to player $i$ as pivotal for $C$ if $d_{G}(C, i)>0$. A power index measures

2. See the work of Chalkiadakis et al. (2011, Thm. 4.2) for why nonnegative integer weights and quotas may be assumed. 
a player's influence in a simple game. Banzhaf III (1965), who rediscovered a notion originally introduced by Penrose (1946), defined the raw Banzhaf power index by

$$
\operatorname{Banzhaf}^{*}(\mathscr{G}, i)=\sum_{C \subseteq N \backslash\{i\}} d_{\mathscr{G}}(C, i)
$$

for a game $\mathscr{G}=(N, v)$ and a player $i$ in $N$. In a simple game, this indicates the number of coalitions a player is pivotal for. However, since the ratios of these indices are more important than their magnitudes, it is useful to normalize them. In fact, two different ways of normalization have been proposed for the Banzhaf index.

In the original definition of the normalized Banzhaf power index (Banzhaf III, 1965), the raw Banzhaf index of a given player is divided by the sum of all players' raw Banzhaf indices:

$$
\overline{\operatorname{Banzhaf}}(\mathscr{G}, i)=\frac{\operatorname{Banzhaf}^{*}(\mathscr{G}, i)}{\sum_{j=1}^{n} \operatorname{Banzhaf}^{*}(\mathscr{G}, j)},
$$

so that all players' normalized Banzhaf indices add up to one. This index has been analyzed in detail by Dubey and Shapley (1979), who introduce an alternative normalization, which divides the raw Banzhaf index of a given player by the total number of coalitions without that player and which they dub the probabilistic Banzhaf power index:

$$
\operatorname{Banzhaf}(\mathscr{G}, i)=\frac{\operatorname{Banzhaf}^{*}(\mathscr{G}, i)}{2^{n-1}} .
$$

Intuitively, this index measures the probability that a player is pivotal for any possible coalition. Based on a comprehensive analysis comparing various mathematical properties of these two power indices, Dubey and Shapley (1979, p. 102) view the probabilistic Banzhaf index as being "in many respects more natural" than the normalized Banzhaf index. In particular, the probabilistic Banzhaf index satisfies four fundamental axioms: (1) symmetry, ${ }^{3}$ (2) dummy player, ${ }^{4}$ (3) additivity, ${ }^{5}$ and (4) a property we call "valuation." "Neither valuation nor additivity is satisfied by the normalized Banzhaf index. It is beyond the purpose of this paper to give a full explanation of these axioms, but we refer the reader to the work of Dubey and Shapley (1979) for a careful, detailed discussion. As the normalized Banzhaf index lacks their fourth axiom, Dubey and Shapley (1979, Footnote 21) conclude: "This may be taken as an initial sign of trouble with the normalization [of the normalized Banzhaf index]." They also note that the probabilistic Banzhaf index "is better behaved when analyzing convergence" (Dubey \& Shapley, 1979, p. 116). Also, the normalized Banzhaf index is subject to the so-called bloc paradox (see Felsenthal \& Machover, 1995), that is, a player can lose power by taking over another player's weight. For the probabilistic Banzhaf index this paradox doesn't hold. (See Sections 3 and 4 for the computational complexity of this annexation problem.)

On the other hand, the normalized Banzhaf power index has its advantages as well, depending on which setting one considers. Aziz et al. (2011, p. 61) argue that: "Although the probabilistic

3. In each game $\mathscr{G}, \operatorname{Banzhaf}(\mathscr{G}, i)=\operatorname{Banzhaf}(\mathscr{G}, j)$ whenever $i$ and $j$ are symmetric, i.e., $v(C \cup\{i\})=v(C \cup\{j\})$ for all coalitions $C \subseteq N \backslash\{i, j\}$.

4. In each game $\mathscr{G}, \operatorname{Banzhaf}(G, i)=0$ whenever $i$ is a dummy player, i.e., $v(C \cup\{i\})=v(C)$ for each coalition $C \subseteq N$.

5. For any two games $\mathscr{G}_{1}=\left(N, v_{1}\right)$ and $\mathscr{G}_{2}=\left(N, v_{2}\right)$, $\operatorname{Banzhaf}\left(\mathscr{G}_{1}+\mathscr{G}_{2}, i\right)=\operatorname{Banzhaf}\left(\mathscr{G}_{1}, i\right)+\operatorname{Banzhaf}\left(\mathscr{G}_{2}, i\right)$ for all players $i \in N$, where $\mathscr{G}_{1}+\mathscr{G}_{2}=\left(N, v_{1}+v_{2}\right)$ is defined via $\left(v_{1}+v_{2}\right)(C)=v_{1}(C)+v_{2}(C)$ for all coalitions $C \subseteq N$.

6. For any two simple games $\mathscr{G}_{1}=\left(N, v_{1}\right)$ and $\mathscr{G}_{2}=\left(N, v_{2}\right)$, it holds that $\operatorname{Banzhaf}\left(\mathscr{G}_{v_{1} \vee v_{2}}, i\right)+\operatorname{Banzhaf}\left(\mathscr{G}_{v_{1} \wedge v_{2}}, i\right)=$ $\operatorname{Banzhaf}\left(\mathscr{G}_{1}, i\right)+\operatorname{Banzhaf}\left(\mathscr{G}_{2}, i\right)$ for each $i \in N$, where the games $\mathscr{G}_{v_{1} \vee v_{2}}=\left(N, v_{1} \vee v_{2}\right)$ and $\mathscr{G}_{v_{1} \wedge v_{2}}=\left(N, v_{1} \wedge v_{2}\right)$ are defined by $\left(v_{1} \vee v_{2}\right)(C)=\max \left(v_{1}(C), v_{2}(C)\right)$ and $\left(v_{1} \wedge v_{2}\right)(C)=\min \left(v_{1}(C), v_{2}(C)\right)$ for all coalitions $C \subseteq N$. 
Banzhaf index is more useful measuring the actual probability of influencing a decision, it does not fit the framework of using power indices to share resources or power, because the probabilistic Banzhaf index is not normalized." Here, normalization is done with respect to the number of players/coalitions, which makes games with different numbers of players better comparable. Due to its normalization with respect to all players in a game, in monotonic games the normalized Banzhaf index yields an imputation (i.e., a payoff vector $(\overline{\operatorname{Banzhaf}}(\mathscr{G}, 1), \overline{\operatorname{Banzhaf}}(\mathscr{G}, 2), \ldots, \overline{\operatorname{Banzhaf}}(\mathscr{G}, n))$ satisfying efficiency, $\sum_{i=1}^{n} \overline{\operatorname{Banzhaf}}(\mathscr{G}, i)=v(N)$, and individual rationality, $\overline{\operatorname{Banzhaf}}(\mathscr{G}, i) \geq v(\{i\})$ for all $i \in N)$, whereas the probabilistic Banzhaf index is not efficient.

There is a unique imputation satisfying all four axioms mentioned above, based on the ShapleyShubik power index (Shapley \& Shubik, 1954), which describes the marginal contributions of a player to all possible coalitions with respect to the order in which players enter the coalitions:

$$
\text { ShapleyShubik }^{*}(\mathscr{G}, i)=\sum_{C \subseteq N \backslash\{i\}}\|C\| ! \cdot(n-1-\|C\|) ! \cdot d_{\mathscr{G}}(C, i),
$$

normalized by

$$
\operatorname{ShapleyShubik}(\mathscr{G}, i)=\frac{\operatorname{ShapleyShubik}^{*}(\mathscr{G}, i)}{n !} .
$$

For coalitional games, this is also known as the Shapley value (Shapley, 1953).

Felsenthal and Machover $(2005,1995)$ carefully discuss the differences between these power indices, and we refer the reader to their work.

\subsection{Basic Notions from Computational Complexity Theory}

We assume that the reader is familiar with the basic notions of complexity theory such as the complexity classes P (“deterministic polynomial time") and NP ("nondeterministic polynomial time"), the polynomial-time many-one reducibility, denoted by $\leq_{\mathrm{m}}^{\mathrm{p}}$, and the notions of hardness and completeness (of decision problems for complexity classes) with respect to $\leq_{\mathrm{m}}^{\mathrm{p}}$. For more background and details, see, e.g., the textbooks by Garey and Johnson (1979), Papadimitriou (1995), and Rothe (2005).

Valiant (1979) introduces \#P as the class of functions that give the number of solutions of the instances of NP problems. For a decision problem $A \in \mathrm{NP}$, we denote this function by \#A. For example, letting SAT denote the satisfiability problem from propositional logic, \#S AT denotes the function mapping any boolean formula $\varphi$ to the number of truth assignments satisfying $\varphi$. There are several notions of reducibility for functional problems and, consequently, there are several notions of hardness and completeness for complexity classes of functions such as \#P. Let $f$ and $g$ be two functions mapping from $\Sigma^{*}$ to $\mathbb{N}$. We say $f$ many-one-reduces to $g$ if there exist two polynomialtime computable functions, $\varphi$ and $\psi$, such that for each $x \in \Sigma^{*}, f(x)=\psi(g(\varphi(x)))$. This notion of functional many-one reducibility is due to Zankó (1991); it is the analogue of (polynomial-time) many-one reducibility for sets. The special case where $\psi$ is the identity yields the parsimonious reducibility, which preserves the number of solutions: We say $f$ parsimoniously reduces to $g$ if there exists a polynomial-time computable function $\varphi$ such that for each $x \in \Sigma^{*}, f(x)=g(\varphi(x))$. Intuitively, parsimonious reductions preserve the number of solutions (for a more detailed discussion of functional reducibilities see Faliszewski \& Hemaspaandra, 2009). We say $g$ is \#P-parsimonioushard if every function $f \in \# \mathrm{P}$ parsimoniously reduces to $g$. If $g$ is \#P-parsimonious-hard and $g \in$ \#P, then $g$ is \#P-parsimonious-complete. The notions of \#P-many-one-hardness and \#P-many-onecompleteness are defined analogously. It is known that, given a WVG $\mathscr{G}$ and a player $i$, computing 
the raw Banzhaf index is \#P-parsimonious-complete (Prasad \& Kelly, 1990), whereas computing the raw Shapley-Shubik index is not (Faliszewski \& Hemaspaandra, 2009), although it is \#P-manyone-complete. For other recent \#P-completeness results, we refer to the work of Aziz, Brandt, and Brill (2013).

Gill (1977) introduces the class PP ("probabilistic polynomial time") that contains all decision problems $X$ for which there exist a function $f \in \# \mathrm{P}$ and a polynomial $p$ such that for all instances $x$, $x \in X$ if and only if $f(x) \geq 2^{p(|x|)-1}$. It is easy to see that NP $\subseteq$ PP; in fact, PP is considered to be by far a larger class than NP, due to Toda's theorem (1991): PP is at least as hard (in terms of polynomial-time Turing reductions) as any problem in the polynomial hierarchy (i.e., $\mathrm{PH} \subseteq \mathrm{P}^{\mathrm{PP}}$ ). $\mathrm{NP}^{\mathrm{PP}}$, the second level of Wagner's counting hierarchy (1986), is the class of problems solvable by an NP machine with access to a PP oracle. Mundhenk, Goldsmith, Lusena, and Allender (2000) identify $\mathrm{NP}^{\mathrm{PP}}$-complete problems related to finite-horizon Markov decision processes. Littman, Goldsmith, and Mundhenk (1998) obtain $\mathrm{NP}^{\mathrm{PP}}$-completeness results when analyzing a variant of the satisfiability problem and questions related to probabilistic planning.

\section{Definition of Beneficial Merging, Splitting, and Annexation}

Aziz et al. (2011) introduce the merging and splitting operations for WVGs. We use the following notation. Given a WVG $\mathscr{G}=\left(w_{1}, \ldots, w_{n} ; q\right)$ and a nonempty ${ }^{7}$ coalition $S \subseteq\{1, \ldots, n\}$, let $\mathscr{G}_{\& S}=$ $\left(w(S), w_{j_{1}}, \ldots, w_{j_{n-\|S\|}} ; q\right)$ with $\left\{j_{1}, \ldots, j_{n-\|S\|}\right\}=N \backslash S$ denote the new WVG in which the players in $S$ have been merged into one new player of weight $w(S) .{ }^{8}$ For a power index PI, the beneficial merging problem is defined as follows.

\begin{tabular}{ll}
\hline & \multicolumn{1}{c}{ PI-BENEFICIALMERGE } \\
\hline Given: & A WVG $\mathscr{G}=\left(w_{1}, \ldots, w_{n} ; q\right)$ and a nonempty coalition $S \subseteq\{1, \ldots, n\}$. \\
Question: & Is it true that $\operatorname{PI}\left(\mathscr{G}_{\& S}, 1\right)>\sum_{i \in S} \operatorname{PI}(\mathscr{G}, i) ?$ \\
\hline
\end{tabular}

Similarly, given a WVG $\mathscr{G}=\left(w_{1}, \ldots, w_{n} ; q\right)$, a player $i$, and an integer $m \geq 2$, define the set of WVGs $\mathscr{G}_{i \div m}=\left(w_{1}, \ldots, w_{i-1}, w_{i+1}, \ldots, w_{n}, w_{n+1}, \ldots, w_{n+m} ; q\right)$ in which $i$ with weight $w_{i}$ is split into $m$ new players $n+1, \ldots, n+m$ with weights $w_{n+1}, \ldots, w_{n+m}$ such that $\sum_{j=1}^{m} w_{n+j}=w_{i}$. (Note that there is a set of such WVGs $\mathscr{G}_{i \div m}$, since there might be several possibilities of distributing $i$ 's weight $w_{i}$ to the new players $n+1, \ldots, n+m$ satisfying $\sum_{j=1}^{m} w_{n+j}=w_{i}$.)

We distinguish between two different splitting problems. ${ }^{9}$ First, for a power index PI, consider the problem where a weighted voting game, a player $i$, and the number $m$ of false identities $i$ splits into are given in the problem instance, but not the weights of the new players:

\section{PI-BENEFICIALSPLIT}

Given: $\quad \operatorname{A~WVG} \mathscr{G}=\left(w_{1}, \ldots, w_{n} ; q\right)$, a player $i$, and an integer $m \geq 2$.

Question: Is it possible to split $i$ into $m$ new players $n+1, \ldots, n+m$ with weights $w_{n+1}, \ldots, w_{n+m}$ satisfying $\sum_{j=1}^{m} w_{n+j}=w_{i}$ such that in this new WVG, call it $\mathscr{G}_{i \div m}$, it holds that $\sum_{j=1}^{m} \operatorname{PI}\left(\mathscr{G}_{i \div m}, n+j\right)>\operatorname{PI}(\mathscr{G}, i)$ ?

7. We omit the empty coalition, since this would slightly change the idea of the problem.

8. Note that the players' order doesn't matter when considering the normalized or probabilistic Banzhaf index.

9. This distinction wouldn't make sense for beneficial merging or annexation. 
As mentioned above, for an instance $(\mathscr{G}, i, m)$ of PI-BENEFICIALSPLIT, there might be various ways of distributing $i$ 's weight to her false identities, giving rise to various new games $\mathscr{G}_{i \div m}$. In the second (more special) variant of the problem we consider, the new players' weights are given explicitly in the problem instance (and thus the number of false identities is given implicitly). In this case, there is only one unique new game $\mathscr{G}_{i \div m}$, and splitting is the inverse function to merging. We will use PI-BENEFICIALSPLIT to denote the more general problem without explicitly given weights, and will explicitly mention it whenever we speak of the more restricted variant.

While these problems deal with voluntary actions by players, Felsenthal and Machover (1995) study the question of whether it is possible for a player to change her power by taking another player's weight over without this player's consent. They introduce the bloc paradox, stating that it is possible to lose power by annexing another player's weight. For instance, the normalized Banzhaf index is subject to this paradox. For the Shapley-Shubik index, Felsenthal and Machover show that annexation is never disadvantageous. Nevertheless, one can still ask the question of whether it is in fact advantageous. The following problem has been studied by Aziz et al. (2011). Let PI be a power index.

\begin{tabular}{ll}
\hline & \multicolumn{1}{c}{ PI-BEnEFICIALANNEXATION } \\
\hline Given: & A WVG $\mathscr{G}=\left(w_{1}, \ldots, w_{n} ; q\right)$, a player $i \in N$, and a coalition $S \subset\{1, \ldots, n\} \backslash\{i\}$. \\
Question: & Is it true that $\operatorname{PI}\left(\mathscr{G}_{\&(S \cup\{i\})}, 1\right)>\operatorname{PI}(\mathscr{G}, i) ?$ \\
\hline
\end{tabular}

In particular, Aziz et al. (2011) show that it is NP-hard to decide whether annexation is beneficial for the normalized Banzhaf index, and that with respect to this power index it is always beneficial for a player to annex another player with a larger weight. They also introduce the annexation nonmonotonicity paradox, which says that it sometimes can be more useful for a player to annex another player of small weight than to annex another player of large weight.

The goal of this paper is to classify these problems in terms of their complexity for both the Shapley-Shubik and the probabilistic Banzhaf index. First, since we allow players with zero weight, the analysis of the beneficial splitting problem requires another simple fact, which we will use in the upcoming proofs of Theorems 4.7 and 4.12.

Lemma 3.1. For both the probabilistic Banzhaf index and the Shapley-Shubik index, given a weighted voting game, adding a player with weight zero does not change the original players' power indices, and the new player's power index is zero.

The proof of Lemma 3.1 is straightforward and therefore omitted.

\section{Complexity of Beneficial Merging, Splitting, and Annexation in Weighted Voting Games}

Aziz et al. (2011) analyze the problems ShapleyShubik-BENEFICIALMERGEand ShapleyShubikBENEFICIALSPLIT as well as Banzhaf-BENEFICIALMERGE and Banzhaf-BENEFICIALSPLiT in terms of their complexity. They provide NP-hardness lower bounds, but leave it open whether these problems are in NP. Indeed, Aziz et al. (2011, p. 72, Remark 13) note that "it is quite possible that our problems are not in NP," which raises a natural question: What about the upper bounds? Faliszewski and Hemaspaandra (2009) establish an upper bound for the restriction of the beneficial merging problem for weighted voting games that was originally proposed by Bachrach and Elkind 
(2008) (“Can any two players increase their joint Shapley-Shubik index via merging?"): This problem is in the complexity class PP. In that paper, Faliszewski and Hemaspaandra study the problem PI-POWERCOMPARE in weighted voting games, where PI is some power index. They prove PPcompleteness of this problem for the probabilistic Banzhaf and the Shapley-Shubik index.

In this section we prove that beneficial merging and splitting is PP-hard, and we provide matching upper bounds for beneficial merging and splitting (the latter in the variant where the new players' weights are given), both for the Shapley-Shubik and the probabilistic Banzhaf index.

\subsection{The Probabilistic Banzhaf Power Index}

Both the beneficial merging problem for a coalition $S$ of size 2 and the beneficial splitting problem for $m=2$ false identities can trivially be decided in polynomial time for the probabilistic Banzhaf index, since the sum of power (in terms of this index) of two players is always equal to the power of the player that is obtained by merging them.

Proposition 4.1. Let $\mathscr{G}$ be a weighted voting game and $S \subseteq\{1, \ldots, n\}$ be a coalition of its players.

1. Banzhaf-BeneficialMerge is in $\mathrm{P}$ for instances $(\mathscr{G}, S)$ with $\|S\|=2$.

2. Banzhaf-Beneficial Split is in $\mathrm{P}$ for instances $(\mathscr{G}, i, 2)$.

Proof. Let $\mathscr{G}=\left(w_{1}, \ldots, w_{n} ; q\right)$ be a weighted voting game. Without loss of generality (see Footnote 8$)$, let $S=\{1, n\}$. We obtain a new game $\mathscr{G}_{\& S}=\left(w_{1}+w_{n}, w_{2}, \ldots, w_{n-1} ; q\right)$, where the first player is the new player merging $S$. Letting $v_{\mathscr{G}}$ and $v_{\mathscr{G}_{\& S}}$ denote the corresponding coalitional functions, it holds that

$$
\begin{aligned}
\operatorname{Banzhaf}\left(\mathscr{G}_{\& S}, 1\right)-(\operatorname{Banzhaf}(\mathscr{G}, 1)+\operatorname{Banzhaf}(\mathscr{G}, n)) & \frac{1}{2^{n-2}}\left(\sum_{C \subseteq\{2, \ldots, n-1\}}\left(v_{\mathscr{G}_{\& S}}(C \cup\{1\})-v_{\mathscr{G}_{\& S}}(C)\right)\right) \\
& -\frac{1}{2^{n-1}}\left(\sum_{C \subseteq\{2, \ldots, n\}}\left(v_{\mathscr{G}}(C \cup\{1\})-v_{\mathscr{G}}(C)\right)+\sum_{C \subseteq\{1, \ldots, n-1\}}\left(v_{\mathscr{G}}(C \cup\{n\})-v_{\mathscr{G}}(C)\right)\right) \\
= & \frac{1}{2^{n-1}}\left(\sum _ { C \subseteq \{ 2 , \ldots , n - 1 \} } \left(2\left(v_{\mathscr{G}_{\mathscr{E}}}(C \cup\{1\})-v_{\mathscr{G}_{\mathscr{G}}}(C)\right)\right.\right. \\
-\left(v_{\mathscr{G}}(C \cup\{1\})-v_{\mathscr{G}}(C)\right)-\left(v_{\mathscr{G}}(C \cup\{1, n\})-v_{\mathscr{G}}(C \cup\{n\})\right) & \left.\left.-\left(v_{\mathscr{G}}(C \cup\{n\})-v_{\mathscr{G}}(C)\right)-\left(v_{\mathscr{G}}(C \cup\{n, 1\})-v_{\mathscr{G}}(C \cup\{1\})\right)\right)\right) \\
= & \frac{1}{2^{n-1}}\left(\sum_{C \subseteq\{2, \ldots, n-1\}}\left(2 v_{\mathscr{G}_{\mathscr{S} S}}(C \cup\{1\})-2 v_{\mathscr{G}}(C \cup\{1, n\})+2 v_{\mathscr{G}}(C)-2 v_{\mathscr{G}}(C)\right)\right)=0 .
\end{aligned}
$$

In the case of splitting, it similarly holds that

$$
\operatorname{Banzhaf}\left(\mathscr{G}_{n \div 2}, n+1\right)+\operatorname{Banzhaf}\left(\mathscr{G}_{n \div 2}, n+2\right)-\operatorname{Banzhaf}(\mathscr{G}, n)=0
$$


for a weighted voting game $\mathscr{G}=(N, v), m=2$, and, without loss of generality, player $n$ in $\mathscr{G}$ splitting into players $n+1$ and $n+2$ in a new game $\mathscr{G}_{n \div 2}$.

Although it may seem as if Proposition 4.1 implied that merging (and splitting) were never beneficial regarding this index, this cannot be generalized to merging (or splitting into) more than two players, by repeatedly applying the above result to pairs of players step by step. For example, as soon as two players merge, a third player's probabilistic Banzhaf index might have already changed in the new game, before merging her with another player in a subsequent step. Suppose three players $\{1,2,3\}$ want to merge in a game $\mathscr{G}$. Let $B_{i}=\operatorname{Banzhaf}(\mathscr{G}, i), 1 \leq i \leq 3$, be their original probabilistic Banzhaf indices. Let $B$ be their common Banzhaf index after the merge. After merging the first two players, let $B_{1}^{\prime}$ and $B_{3}^{\prime}$ be the indices of $\{1,2\}$ and 3, respectively. Then, due to Proposition 4.1, $B=$ $B_{1}^{\prime}+B_{3}^{\prime}=B_{1}+B_{2}+B_{3}^{\prime}$. Hence, $B>B_{1}+B_{2}+B_{3}$ if and only if $B_{3}^{\prime}>B_{3}$. That is, for the probabilistic Banzhaf index, beneficial merging of three players boils down to comparing the index of one player in two games - the original game and the one where the other two players have merged. If these were two arbitrary games, the result for PI-POWERCOMPARE by Faliszewski and Hemaspaandra (2009) would have applied. Here, however, the indices need to be compared in two closely related games; this requires a different proof. Indeed, next we show that it is by far harder (unless the polynomial hierarchy collapses to its first level) to decide whether merging three players is beneficial in terms of the probabilistic Banzhaf index than for two players. We will use the following result due to Faliszewski and Hemaspaandra (2009, Lemma 2.3).

Lemma 4.2 (Faliszewski \& Hemaspaandra, 2009). Let $F$ be a \#P-parsimonious-complete function. The problem COMPARE- $F=\{(x, y) \mid F(x)>F(y)\}$ is PP-complete.

The well-known NP-complete problem SUBSETSUM (which is a special variant of the KNAPSACK problem) asks, given a sequence $\left(a_{1}, \ldots, a_{n}\right)$ of positive integers and a positive integer $q$, do there exist $x_{1}, \ldots, x_{n} \in\{0,1\}$ such that $\sum_{i=1}^{n} x_{i} a_{i}=q$ ? It is known that \#SUBSETSUM is \#Pparsimonious-complete (for parsimonious reductions from \#3-SAT via \#EXACTCOVERBY3-SETS to \#SUBSETSum see, e.g., Hunt, Marathe, Radhakrishnan, \& Stearns, 1998; Papadimitriou, 1995). Hence, by Lemma 4.2, we have the following.

Corollary 4.3. COMPARE-\#SUBSETSUM is PP-complete.

Our goal is to provide a $\leq_{\mathrm{m}}^{\mathrm{p}}$-reduction from COMPARE-\#SUBSETSUM to Banzhaf-BENEFICIALMerge. However, to make this reduction work, it will be useful to consider two restricted variants of COMPARE-\#SUBSETSum, which we denote by COMPARE-\#SUBSETSUM-R and COMPARE-\#SUBSETSUM-RR, show their PP-hardness, and then reduce COMPARE-\#SUBSETSUM-RR to Banzhaf-BeneficialMerge. This will be done in Lemmas 4.4 and 4.5 and in Theorem 4.6. In all restricted variants of COMPARE-\#SUBSETSUM we may assume, without loss of generality, that the target value $q$ in a related \#SUBSETSUM instance $\left(\left(a_{1}, \ldots, a_{n}\right), q\right)$ satisfies $1 \leq q \leq \alpha-1$, where $\alpha=\sum_{i=1}^{n} a_{i}$. 
COMPARE-\#SUBSETSUM-R

Given: $\quad$ A sequence $A=\left(a_{1}, \ldots, a_{n}\right)$ of positive integers and two positive integers $q_{1}$ and $q_{2}$ with $1 \leq q_{1}, q_{2} \leq \alpha-1$, where $\alpha=\sum_{i=1}^{n} a_{i}$.

Question: Is the number of subsequences of $A$ summing up to $q_{1}$ greater than the number of subsequences of $A$ summing up to $q_{2}$, that is, does it hold that

\#SUbSetSum $\left(\left(a_{1}, \ldots, a_{n}\right), q_{1}\right)>$ \#SUBSETSUM $\left(\left(a_{1}, \ldots, a_{n}\right), q_{2}\right)$ ?

\section{Lemma 4.4. COMPARE-\#SUBSETSUM $\leq_{m}^{\mathrm{p}}$ COMPARE-\#SUBSETSUM-R.}

Proof. Given an instance $(X, Y)$ of Compare-\#SubsetSum, $X=\left(\left(x_{1}, \ldots, x_{m}\right), q_{x}\right)$ and $Y=$ $\left(\left(y_{1}, \ldots, y_{n}\right), q_{y}\right)$, construct a COMPARE-\#SUBSETSUM-R instance $\left(A, q_{1}, q_{2}\right)$ as follows. Let $\alpha=$ $\sum_{i=1}^{m} x_{i}$ and define $A=\left(x_{1}, \ldots, x_{m}, 2 \alpha y_{1}, \ldots, 2 \alpha y_{n}\right), q_{1}=q_{x}$, and $q_{2}=2 \alpha q_{y}$. This construction can obviously be achieved in polynomial time.

It holds that integers from $A$ can only sum up to $q_{x} \leq \alpha-1$ if they do not contain multiples of

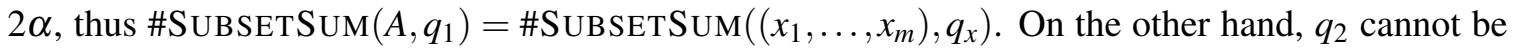
obtained by adding any of the $x_{i}$ 's, since this would yield a non-zero remainder modulo $2 \alpha$, because $\sum_{i=1}^{m} x_{i}=\alpha$ is too small. Thus, it holds that \#SUbSetSum $\left(A, q_{2}\right)=\# \operatorname{SUBSETSUM}\left(\left(y_{1}, \ldots, y_{n}\right), q_{y}\right)$. It follows that $(X, Y)$ is in COMPARE-\#SUBSETSUM if and only if $\left(A, q_{1}, q_{2}\right)$ is in COMPARE\#SUBSETSUM-R.

In order to perform the next step, we need to ensure that all integers in a COMPARE-\#SUBSETSuM-R instance are divisible by 8 . This can easily be achieved, by multiplying each integer in an instance $\left(\left(a_{1}, \ldots, a_{n}\right), q_{1}, q_{2}\right)$ by 8 , obtaining $\left(\left(8 a_{1}, \ldots, 8 a_{n}\right), 8 q_{1}, 8 q_{2}\right)$ without changing the number of solutions for both related SUBSETSUM instances. Thus, from now on, without loss of generality, we assume that for a given COMPARE-\#SUBSETSUM-R instance $\left(\left(a_{1}, \ldots, a_{n}\right), q_{1}, q_{2}\right)$, it holds that $a_{i}, q_{j} \equiv 0 \bmod 8$ for $1 \leq i \leq n$ and $j \in\{1,2\}$.

Now, we consider our even more restricted variant of this problem.

\begin{tabular}{ll}
\hline \multicolumn{1}{c}{ COMPARE-\#SUBSETSUM-RR } \\
\hline Given: & A sequence $A=\left(a_{1}, \ldots, a_{n}\right)$ of positive integers. \\
Question: & $\begin{array}{l}\text { Is the number of subsequences of } A \text { summing up to }(\alpha / 2)-2, \text { where } \alpha=\sum_{i=1}^{n} a_{i}, \text { greater } \\
\text { than the number of subsequences of } A \text { summing up to }(\alpha / 2)-1, \text { i.e., is it true that } \\
\\
\text { \#SUBSETSUM }\left(\left(a_{1}, \ldots, a_{n}\right),(\alpha / 2)-2\right)>\text { \#SUBSETSUM }\left(\left(a_{1}, \ldots, a_{n}\right),(\alpha / 2)-1\right) ?\end{array}$ \\
\hline
\end{tabular}

\section{Lemma 4.5. COMPARE-\#SUBSETSUM-R $\leq_{\mathrm{m}}^{\mathrm{p}}$ COMPARE-\#SUBSETSUM-RR.}

Proof. Given an instance $\left(A, q_{1}, q_{2}\right)$ of COMPARE-\#SUbSETSUM-R, where we assume that $A=$ $\left(a_{1}, \ldots, a_{n}\right), q_{1}$, and $q_{2}$ satisfy $a_{i}, q_{j} \equiv 0 \bmod 8$ for $1 \leq i \leq n$ and $j \in\{1,2\}$, we construct an instance $B$ of COMPARE-\#SUBSETSUM-RR as follows. (This reduction is inspired by the standard reduction from SubSETSUM to PARTITION due to Karp, 1972.)

Letting $\alpha=\sum_{i=1}^{n} a_{i}$, define

$$
B=\left(a_{1}, \ldots, a_{n}, 2 \alpha-q_{1}, 2 \alpha+1-q_{2}, 2 \alpha+3+q_{1}+q_{2}, 3 \alpha\right) .
$$


This instance can obviously be constructed in polynomial time. Observe that

$$
T=\left(\sum_{i=1}^{n} a_{i}\right)+\left(2 \alpha-q_{1}\right)+\left(2 \alpha+1-q_{2}\right)+\left(2 \alpha+3+q_{1}+q_{2}\right)+3 \alpha=10 \alpha+4,
$$

and therefore, $(T / 2)-2=5 \alpha$ and $(T / 2)-1=5 \alpha+1$. We show that $\left(A, q_{1}, q_{2}\right)$ is in COMPARE-\#SUBSETSUM-R if and only if $B$ is in COMPARE-\#SUBSETSUM-RR.

First, we examine which subsequences of $B$ sum up to $5 \alpha$. Consider two cases.

Case 1: If $3 \alpha$ is added, $2 \alpha+3+q_{1}+q_{2}$ cannot be added, as it would be too large. Also, $2 \alpha+1-q_{2}$ cannot be added, leading to an odd sum. So, $2 \alpha-q_{1}$ has to be added, as the remaining $\alpha$ are too small. Since $3 \alpha+2 \alpha-q_{1}=5 \alpha-q_{1}, 5 \alpha$ can be achieved by adding some $a_{i}$ 's if and only if there exists a subset $A^{\prime} \subseteq\{1, \ldots, n\}$ such that $\sum_{i \in A^{\prime}} a_{i}=q_{1}$ (i.e., $A^{\prime}$ is a solution of the SUbSETSum instance $\left.\left(A, q_{1}\right)\right)$.

Case 2: If $3 \alpha$ is not added, but $2 \alpha+3+q_{1}+q_{2}$, an even number can only be achieved by adding $2 \alpha+1-q_{2}$, thus, $\alpha-4-q_{1}$ remain. $2 \alpha-q_{1}$ is too large, while no subsequence of $A$ sums up to $\alpha-4-q_{1}$, because of the assumption of divisibility by 8 . If neither $3 \alpha$ nor $2 \alpha+3+q_{1}+q_{2}$ are added, the remaining $5 \alpha+1-q_{1}-q_{2}$ are too small.

Thus, the only possibility to obtain $5 \alpha$ is to find a subsequence of $A$ adding up to $q_{1}$. Thus, \#SubSetSum $\left(A, q_{1}\right)=\# \operatorname{SubSETSUM}(B, 5 \alpha)$.

Second, for similar reasons, a sum of $5 \alpha+1$ can only be achieved by adding $3 \alpha+(2 \alpha+$ $\left.1-q_{2}\right)$ and a term $\sum_{i \in A^{\prime}} a_{i}$, where $A^{\prime}$ is a subset of $\{1, \ldots, n\}$ such that $\sum_{i \in A^{\prime}} a_{i}=q_{2}$. Hence, \#SUbSETSum $\left(A, q_{2}\right)=\# \operatorname{SubSETSUM}(B, 5 \alpha+1)$.

Thus, the relation \#SUbSETSUM $\left(A, q_{1}\right)>$ \#SUbSETSUM $\left(A, q_{2}\right)$ holds if and only if \#SUBSET$\operatorname{Sum}(B, 5 \alpha)>\# \operatorname{SUBSETSUM}(B, 5 \alpha+1)$, which completes the proof.

We now are ready to prove the main theorem of this section.

Theorem 4.6. Banzhaf-BEnEFICIALMERGE is PP-complete, even if only three players of equal weight merge.

Proof. Membership of Banzhaf-BeneficialMerge in PP follows from the fact that the raw Banzhaf index is in \#P and that \#P is closed under addition and multiplication by two, ${ }^{10}$ and, furthermore, since comparing the values of two \#P functions on two (possibly different) inputs reduces to a PP-complete problem. This technique (which was proposed by Faliszewski \& Hemaspaandra, 2009, and applies their Lemma 2.10) works, since PP is closed under $\leq_{\mathrm{m}}^{\mathrm{p}}$-reducibility.

We show PP-hardness of Banzhaf-BENEFICIALMERGE by means of $\mathrm{a} \leq_{\mathrm{m}}^{\mathrm{p}}$-reduction from COMPARE-\#SUBSETSUM-RR, which is PP-hard by Corollary 4.3 via Lemmas 4.4 and 4.5.

Given an instance $A=\left(a_{1}, \ldots, a_{n}\right)$ of COMPARE-\#SUBSETSUM-RR, construct the following instance for Banzhaf-BeneficialMERGE. Let $\alpha=\sum_{i=1}^{n} a_{i}$. Define the WVG

$$
\mathscr{G}=\left(2 a_{1}, \ldots, 2 a_{n}, 1,1,1,1 ; \alpha\right)
$$

10. Again, note that this idea cannot be transferred straightforwardly to the normalized Banzhaf index, since in different games the indices have possibly different denominators, not only different by a factor of some power of two, as is the case for the probabilistic Banzhaf index. 
with $n+4$ players, and let the merging coalition be $S=\{n+2, n+3, n+4\}$.

Letting $N=\{1, \ldots, n\}$, it holds that

$$
\begin{aligned}
& \text { Banzhaf }(\mathscr{G}, n+2)=\frac{1}{2^{n+3}}\left\|\left\{C \subseteq\{1, \ldots, n+1, n+3, n+4\} \mid \sum_{i \in C} w_{i}=\alpha-1\right\}\right\| \\
& =\frac{1}{2^{n+3}}\left(\left\|\left\{A^{\prime} \subseteq N \mid \sum_{i \in A^{\prime}} 2 a_{i}=\alpha-1\right\}\right\|+3 \cdot\left\|\left\{A^{\prime} \subseteq N \mid 1+\sum_{i \in A^{\prime}} 2 a_{i}=\alpha-1\right\}\right\|\right. \\
& \left.+3 \cdot\left\|\left\{A^{\prime} \subseteq N \mid 2+\sum_{i \in A^{\prime}} 2 a_{i}=\alpha-1\right\}\right\|+\left\|\left\{A^{\prime} \subseteq N \mid 3+\sum_{i \in A^{\prime}} 2 a_{i}=\alpha-1\right\}\right\|\right) \\
& =\frac{1}{2^{n+3}}\left(3 \cdot\left\|\left\{A^{\prime} \subseteq N \mid \sum_{i \in A^{\prime}} 2 a_{i}=\alpha-2\right\}\right\|+\left\|\left\{A^{\prime} \subseteq N \mid \sum_{i \in A^{\prime}} 2 a_{i}=\alpha-4\right\}\right\|\right),
\end{aligned}
$$

since the $2 a_{i}$ 's can only add up to an even number. The first of the four sets in (1) and (2) refers to those coalitions that do not contain any of the players $n+1, n+3$, and $n+4$; the second, third, and fourth set in (1) and (2) refers to those coalitions containing either one, two, or three of them, respectively. Since the players in $S$ have the same weight, players $n+3$ and $n+4$ have the same probabilistic Banzhaf index as player $n+2$.

Furthermore, the new game after merging is $\mathscr{G}_{\&\{n+2, n+3, n+4\}}=\left(3,2 a_{1}, \ldots 2 a_{n}, 1 ; \alpha\right)$ with $n+2$ players, and similarly as above the Banzhaf index of the first player is calculated as follows:

$$
\begin{aligned}
& \text { Banzhaf }\left(\mathscr{G}_{\&\{n+2, n+3, n+4\}}, 1\right) \\
& =\frac{1}{2^{n+1}}\left\|\left\{C \subseteq\{2, \ldots, n+2\} \mid \sum_{i \in C} w_{i} \in\{\alpha-3, \alpha-2, \alpha-1\}\right\}\right\| \\
& =\frac{1}{2^{n+1}}\left(\left\|\left\{A^{\prime} \subseteq N \mid \sum_{i \in A^{\prime}} 2 a_{i} \in\{\alpha-3, \alpha-2, \alpha-1\}\right\}\right\|\right. \\
& \left.\quad+\left\|\left\{A^{\prime} \subseteq N \mid 1+\sum_{i \in A^{\prime}} 2 a_{i} \in\{\alpha-3, \alpha-2, \alpha-1\}\right\}\right\|\right) \\
& =\frac{1}{2^{n+1}}\left(2 \cdot\left\|\left\{A^{\prime} \subseteq N \mid \sum_{i \in A^{\prime}} 2 a_{i}=\alpha-2\right\}\right\|+\left\|\left\{A^{\prime} \subseteq N \mid \sum_{i \in A^{\prime}} 2 a_{i}=\alpha-4\right\}\right\|\right) .
\end{aligned}
$$

Altogether, it holds that

$$
\begin{aligned}
& \operatorname{Banzhaf}\left(\mathscr{G}_{\&\{n+2, n+3, n+4\}}, 1\right)-\sum_{i \in\{n+2, n+3, n+4\}} \operatorname{Banzhaf}(\mathscr{G}, i) \\
& =\frac{1}{2^{n+1}}\left(2 \cdot\left\|\left\{A^{\prime} \subseteq N \mid \sum_{i \in A^{\prime}} 2 a_{i}=\alpha-2\right\}\right\|+\left\|\left\{A^{\prime} \subseteq N \mid \sum_{i \in A^{\prime}} 2 a_{i}=\alpha-4\right\}\right\|\right) \\
& \quad-\frac{3}{2^{n+3}}\left(3 \cdot\left\|\left\{A^{\prime} \subseteq N \mid \sum_{i \in A^{\prime}} 2 a_{i}=\alpha-2\right\}\right\|+\left\|\left\{A^{\prime} \subseteq N \mid \sum_{i \in A^{\prime}} 2 a_{i}=\alpha-4\right\}\right\|\right)
\end{aligned}
$$




$$
\begin{aligned}
= & \left(\frac{1}{2^{n+1}} \cdot 2-\frac{3}{2^{n+3}} \cdot 3\right)\left\|\left\{A^{\prime} \subseteq N \mid \sum_{i \in A^{\prime}} 2 a_{i}=\alpha-2\right\}\right\| \\
& +\left(\frac{1}{2^{n+1}}-\frac{3}{2^{n+3}}\right)\left\|\left\{A^{\prime} \subseteq N \mid \sum_{i \in A^{\prime}} 2 a_{i}=\alpha-4\right\}\right\| \\
= & -\frac{1}{2^{n+3}} \cdot\left\|\left\{A^{\prime} \subseteq N \mid \sum_{i \in A^{\prime}} a_{i}=\frac{\alpha}{2}-1\right\}\right\|+\frac{1}{2^{n+3}} \cdot\left\|\left\{A^{\prime} \subseteq N \mid \sum_{i \in A^{\prime}} a_{i}=\frac{\alpha}{2}-2\right\}\right\|,
\end{aligned}
$$

which is greater than zero if and only if

$$
\left\|\left\{A^{\prime} \subseteq N \mid \sum_{i \in A^{\prime}} a_{i}=\frac{\alpha}{2}-2\right\}\right\|>\left\|\left\{A^{\prime} \subseteq N \mid \sum_{i \in A^{\prime}} a_{i}=\frac{\alpha}{2}-1\right\}\right\|,
$$

which in turn is the case if and only if the original instance $A$ is in COMPARE-\#SUBSETSUM-RR.

Analogously to the proof of Theorem 4.6, it can be shown that the beneficial splitting problem for at least three false identities with given new weights is PP-complete. However, for the more general beneficial splitting problem where the new players' weights are not given, a PP upper bound cannot be shown straightforwardly. Yet, it can be shown that this problem is PP-hard, even for three false identities.

Theorem 4.7. Banzhaf-BENEFICIALSPLIT is PP-hard (even if the given player can only split into three players of equal weight).

Proof. In order to show PP-hardness for Banzhaf-BENEFICIALSPLIT, we use the same techniques as in Theorem 4.6, appropriately modified. In fact, we will now show PP-hardness for $m=3$ false identities. This result can be expanded to all fixed $m \geq 3$ by splitting into additional players with weight 0 . More precisely, if $m>3$, we consider the same game $\mathscr{G}$ as below and split into three players of weight 1 each and $m-3$ players of weight 0 each. By Lemma 3.1, the sum of all $m$ new players' Banzhaf power is equal to the combined Banzhaf power of the three players. Thus, PP-hardness will hold for splitting into $m>3$ players by essentially the same arguments as given below for splitting into three players.

First, we slightly change the definition of COMPARE-\#SUBSETSUM-RR by switching $(\alpha / 2)-$ 2 and $(\alpha / 2)-1$. The problem (call it COMPARE-\#SUBSETSUM-ЯЯ) of whether the number of subsequences of a given sequence $A$ of positive integers summing up to $(\alpha / 2)-1$ is greater than the number of subsequences of $A$ summing up to $(\alpha / 2)-2$, is PP-hard by the same proof as in Lemma 4.5 with the roles of $q_{1}$ and $q_{2}$ exchanged.

Now, we reduce this problem to Banzhaf-BENEFICIALSPLIT by constructing the following instance of the beneficial splitting problem from an instance $A=\left(a_{1}, \ldots, a_{n}\right)$ of COMPARE-\#SUBSETSUM-ЯЯ. Let $\mathscr{G}=\left(2 a_{1}, \ldots, 2 a_{n}, 1,3 ; \alpha\right)$, where $\alpha=\sum_{j=1}^{n} a_{j}$, and let $i=n+2$ be the player to be split. $\mathscr{G}$ is (apart from the order of players) equivalent to the game obtained by merging in the proof of Theorem 4.6. Thus, letting $N=\{1, \ldots, n\}$, $\operatorname{Banzhaf}(\mathscr{G}, n+2)$ equals

$$
\frac{1}{2^{n+1}}\left(2 \cdot\left\|\left\{A^{\prime} \subseteq N \mid \sum_{j \in A^{\prime}} 2 a_{j}=\alpha-2\right\}\right\|+\left\|\left\{A^{\prime} \subseteq N \mid \sum_{j \in A^{\prime}} 2 a_{j}=\alpha-4\right\}\right\|\right) .
$$


Allowing players with weight zero, there are different possibilities to split player $n+2$ into three players. By Lemma 3.1, splitting $n+2$ into one player with weight 3 and two others with weight 0 is not beneficial. Likewise, splitting $n+2$ into two players with weights 1 and 2 and one player with weight 0 is not beneficial, by Lemma 3.1 and since splitting into two players is not beneficial (by Theorem 1). Thus, the only possibility left is splitting $n+2$ into three players of weight 1 each. This corresponds to the original game in the proof of Theorem 4.6, $\mathscr{G}_{i \div 3}=\left(2 a_{1}, \ldots, 2 a_{n}, 1,1,1,1 ; \alpha\right)$. Therefore,

$$
\begin{aligned}
& \text { Banzhaf }\left(\mathscr{G}_{i \div 3}, n+2\right)=\operatorname{Banzhaf}\left(\mathscr{G}_{i \div 3}, n+3\right)=\operatorname{Banzhaf}\left(\mathscr{G}_{i \div 3}, n+4\right) \\
& \quad=\frac{1}{2^{n+3}}\left(3 \cdot\left\|\left\{A^{\prime} \subseteq N \mid \sum_{j \in A^{\prime}} 2 a_{j}=\alpha-2\right\}\right\|+\left\|\left\{A^{\prime} \subseteq N \mid \sum_{j \in A^{\prime}} 2 a_{j}=\alpha-4\right\}\right\|\right) .
\end{aligned}
$$

Altogether, as in the proof of Theorem 4.6, the sum of the three new players' probabilistic Banzhaf indices minus the probabilistic Banzhaf index of the original player is greater than zero if and only if

$$
\left\|\left\{A^{\prime} \subseteq N \mid \sum_{j \in A^{\prime}} a_{j}=(\alpha / 2)-1\right\}\right\|>\left\|\left\{A^{\prime} \subseteq N \mid \sum_{j \in A^{\prime}} a_{j}=(\alpha / 2)-2\right\}\right\|,
$$

which is true if and only if $A$ is in COMPARE-\#SUBSETSUM-ЯЯ.

Remark 4.8. As an upper bound for the general beneficial splitting problem, we can only show membership in $\mathrm{NP}^{\mathrm{PP}}$, whenever the number of false identities is given in unary, and we conjecture that this problem is even complete for this class. When the number $m$ of false identities but not their weights are given in unary, there are exponentially many possibilities to distribute the split player's weight among her false identities. Nondeterministically guessing such a distribution and then, for each distribution guessed, asking an appropriate PP oracle to check in polynomial time whether their combined Banzhaf power in the new game is greater than the original player's Banzhaf power in the original game, shows that Banzhaf-BENEFICIALSPLIT is in $\mathrm{NP}^{\mathrm{PP}}$.

Whenever the number of false identities is given in the standard binary input format, even this upper bound might no longer be valid.

For a given weighted voting game $\mathscr{G}$ and two players $i$ and $j$ in $\mathscr{G}$, Proposition 4.1 implies that

$$
\operatorname{Banzhaf}\left(\mathscr{G}_{\&\{i, j\}}, 1\right)-\operatorname{Banzhaf}(\mathscr{G}, i)=\operatorname{Banzhaf}(\mathscr{G}, j) \geq 0 .
$$

Therefore, it is never disadvantageous for player $i$ to annex player $j$. Furthermore, we have the following result on the complexity of beneficial annexation for the probabilistic Banzhaf index.

Theorem 4.9. Banzhaf-BENEFICIALANNEXATION is NP-complete for instances $(\mathscr{G}, i, S)$ such that $\|S\|=1$.

Proof. By Equation (3) above, the question of whether the new player's probabilistic Banzhaf index is greater than the original player's probabilistic Banzhaf index is equivalent to the question of whether the annexed player has a positive value in the original game. This property can be decided in nondeterministic polynomial time and is NP-hard by a result due to Prasad and Kelly (1990). 
Remark 4.10. While Banzhaf-BENEFICIALANNEXATION immediately inherits NP-hardness from the special case in Theorem 4.9, that problem's NP upper bound does not generalize straightforwardly.

\subsection{The Shapley-Shubik Power Index}

In order to prove PP-hardness for the merging and splitting problems with respect to the ShapleyShubik index, we need to take a further step back.

EXACT COVER BY 3-SETS (X3C, for short) is another well-known NP-complete decision problem: Given a set $B$ of size $3 k$ and a family $\mathscr{S}$ of subsets of $B$ that have size three each, does there exist a subfamily $\mathscr{S}^{\prime}$ of $\mathscr{S}$ such that $B$ is exactly covered by $\mathscr{S}^{\prime}$ ?

Theorem 4.11. ShapleyShubik-BeneficialMerge is PP-complete, even if only two players of equal weight merge.

Proof. The PP upper bound, which has already been observed for two players by Faliszewski and Hemaspaandra (2009), can be shown analogously to the proof of Theorem 4.6.

For proving the lower bound, observe that the size of a coalition a player is pivotal for is crucial for determining the player's Shapley-Shubik index. Pursuing the techniques by Faliszewski and Hemaspaandra, we examine the problem COMPARE-\#X3C, which is PP-complete by Lemma 4.2. We will apply the following useful properties of X3C instances shown by Faliszewski and Hemaspaandra (2009, Lemma 2.7): Every X3C instance $\left(B^{\prime}, \mathscr{S}^{\prime}\right)$ can be transformed into an X3C instance $(B, \mathscr{S})$, where $\|B\|=3 k$ and $\|\mathscr{S}\|=n$, that satisfies $k / n=2 / 3$ without changing the number of solutions, i.e., $\# \mathrm{X} 3 \mathrm{C}(B, \mathscr{S})=\# \mathrm{X} 3 \mathrm{C}\left(B^{\prime}, \mathscr{S}^{\prime}\right)$. Now, by the properties of the standard reduction from $\mathrm{X} 3 \mathrm{C}$ to SUBSETSUM (which in particular preserves the number of solutions, i.e., \#X3C parsimoniously reduces to \#SUBSETSUM, as well as the "input size" $n$ and the "solution size" $k$, see, e.g., Papadimitriou, 1995), we can assume that in a given COMPARE-\#SUBSETSUM instance each subsequence summing up to the given integer $q$ is of size $2 n / 3$. Following the track of the reductions from COMPARE-\#SUBSETSUM via COMPARE-\#SUBSETSUM-R to COMPARE-\#SUBSETSUM-RR in Lemmas 4.4 and 4.5 , a solution $A^{\prime} \subseteq\{1, \ldots, n\}$ to a given instance $A=\left(a_{1}, \ldots, a_{n}\right)$ of the latter problem $\left(A^{\prime}\right.$ satisfying either $\sum_{i \in A^{\prime}} a_{i}=(\alpha / 2)-2$ or $\sum_{i \in A^{\prime}} a_{i}=(\alpha / 2)-1$, where $\left.\alpha=\sum_{i=1}^{n} a_{i}\right)$ can be assumed to satisfy $\left\|A^{\prime}\right\|=k=(n+2) / 3$. Under this assumption, we show PP-hardness of ShapleyShubik-BENEFICIALMERGE via a reduction from COMPARE-\#SUBSETSUM-RR. Given such an instance, we construct the WVG $\mathscr{G}=\left(a_{1}, \ldots, a_{n}, 1,1 ; \alpha / 2\right)$ and consider coalition $S=\{n+$ $1, n+2\}$. Let $N=\{1, \ldots, n\}$ and define $X=\# \operatorname{SubSETSum}(A,(\alpha / 2)-1)$ and $Y=\# \operatorname{SubSetSum}(A$, $(\alpha / 2)-2)$. Then,

$$
\begin{aligned}
& \text { ShapleyShubik }(\mathscr{G}, n+1)=\operatorname{ShapleyShubik}(\mathscr{G}, n+2) \\
& =\frac{1}{(n+2) !}\left(\left(\sum_{\substack{C \subseteq N \text { such that } \\
\sum_{i \in C} a_{i}=(\alpha / 2)-1}}\|C\| !(n+1-\|C\|) !\right)+\left(\sum_{\substack{C \subseteq N \text { such that } \\
\sum_{i \in C} a_{i}=(\alpha / 2)-2}}(\|C\|+1) !(n-\|C\|) !\right)\right) \\
& =\frac{1}{(n+2) !}(X \cdot k !(n+1-k) !+Y \cdot(k+1) !(n-k) !) .
\end{aligned}
$$


Merging the players in $S$, we obtain $\mathscr{G}_{\& S}=\left(2, a_{1}, \ldots, a_{n} ; \alpha / 2\right)$. The Shapley-Shubik index of the new player in $\mathscr{G}_{\& S}$ is

$$
\begin{aligned}
\text { ShapleyShubik }\left(\mathscr{G}_{\& S}, 1\right) & =\frac{1}{(n+1) !} \sum_{\substack{\sum_{i \in C} a_{i} \in\{(\alpha / 2)-1,(\alpha / 2)-2\} \\
\text { such that }}}\|C\| !(n-\|C\|) ! \\
& =\frac{1}{(n+1) !}(X+Y) \cdot(k+1) !(n-k) !
\end{aligned}
$$

All in all,

$$
\begin{aligned}
& \text { ShapleyShubik }\left(\mathscr{G}_{\& S}, 1\right)-(\operatorname{ShapleyShubik}(\mathscr{G}, n+1)+\operatorname{ShapleyShubik}(\mathscr{G}, n+2)) \\
& =\frac{(X+Y) \cdot(k+1) !(n-k) !}{(n+1) !}-\frac{2(X \cdot k !(n+1-k) !+Y \cdot(k+1) !(n-k) !)}{(n+2) !} \\
& =\frac{k !(n-k) !}{(n+2) !}(n-2 k)(-X+Y) .
\end{aligned}
$$

Since we assumed that $k=(n+2) / 3$ and we can also assume that $n>4$ (because we added four integers in the construction in the proof of Lemma 4.5), it holds that

$$
n-2 k=\frac{n-4}{3}>0 .
$$

Thus the term (4) is greater than zero if and only if $Y$ is greater than $X$, which is true if and only if $A$ is in COMPARE-\#SUBSETSUM-RR.

Analogously to the probabilistic Banzhaf index, we can show that also for the Shapley-Shubik index it is PP-complete to decide if splitting a player into players with given weights is beneficial. For the more general case where the number of false identities but no actual weights are given, we can raise the previously known lower bound to PP-hardness. However, the upper bound of PP cannot be transferred straightforwardly.

Theorem 4.12. ShapleyShubik-BENEFICIALSPLIT is PP-hard (even if the given player can only split into two players of equal weight).

Proof. PP-hardness can be shown analogously to the proof of Theorem 4.7, appropriately modified to use the arguments from the proof of Theorem 4.11 instead of those from the proof of Theorem 4.6.

An upper bound of $\mathrm{NP}^{\mathrm{PP}}$ holds due to analogous arguments as in the proof of Theorem 4.7, whenever $m$ is given in unary.

Felsenthal and Machover (1995) have shown that annexation is never disadvantageous for the Shapley-Shubik index. Still, the question of whether it is advantageous is hard to decide.

Theorem 4.13. ShapleyShubik-BENEFICIALANNEXATION is NP-complete for instances $(\mathscr{G}, i, S)$ with $\|S\|=1$. 
Proof. Let $\mathscr{G}=\left(w_{1}, \ldots, w_{n} ; q\right)$ be a weighted voting game and, without loss of generality, let player 1 annex player $n$. It holds that

$\operatorname{ShapleyShubik}\left(\mathscr{G}_{\&\{1, n\}}, 1\right)-\operatorname{Shapley} \operatorname{Shubik}(\mathscr{G}, 1)$

$$
\begin{aligned}
=\frac{1}{n !} \sum_{C \subseteq\{2, \ldots, n-1\}}((v(C \cup\{1, n\}-v(C \cup\{1\})) \cdot\|C\| !(n-1-\|C\|) ! \\
+(v(C \cup\{n\}-v(C)) \cdot(\|C\|+1) !(n-2-\|C\|) !) .
\end{aligned}
$$

Unlike for the probabilistic Banzhaf index, this term is in general not equal to ShapleyShubik $(\mathscr{G}, n)$, but is greater than zero if and only if player $n$ is pivotal for at least one coalition $C \subseteq\{1, \ldots, n-1\}$ in the original game. So, analogously to Theorem 4.9, this property can be decided in nondeterministic polynomial time and is NP-hard by a result due to Prasad and Kelly (1990) (see also Deng \& Papadimitriou, 1994).

Remark 4.14. Analogously to annexation with respect to the probabilistic Banzhaf index, while ShapleyShubik-BENEFICIALANNEXATION immediately inherits NP-hardness from the special case in Theorem 4.13, that problem's NP upper bound does not generalize straightforwardly.

\section{Generalizing Merging and Splitting Functions}

We extend the definition of merging and splitting functions from weighted voting games to general classes (or representations) $\mathfrak{G}$ of coalitional games; one may think of $\mathfrak{G}$ as being the class of simple games or the family of weighted voting games or any representation of simple games such as the vector weighted voting games (Chalkiadakis et al., 2011), or the threshold network flow games due to Bachrach and Rosenschein (2009), or even the class of all coalitional games.

A merging function on $\mathfrak{G}$,

$$
\mu_{\mathfrak{G}}:\{\mathscr{G}=(N, v) \mid \mathscr{G} \in \mathfrak{G}\} \times(\mathfrak{P}(N) \backslash \emptyset) \rightarrow \mathfrak{G},
$$

turns a given coalitional game $\mathscr{G}=(N, v)$ in suitable representation and a given nonempty coalition $S \subseteq N$ into a new game $\mu_{\mathfrak{G}}(\mathscr{G}, S)=\left(N^{\prime}, v^{\prime}\right)$, where $N^{\prime}=\left\{i_{\& S}\right\} \cup(N \backslash S)$ contains a new player $i_{\& S}$ merging $S$, and $v^{\prime}: \mathfrak{P}\left(N^{\prime}\right) \rightarrow \mathbb{R}$ is the new coalitional function whose values are to be specified according to the type of games in class $\mathfrak{G}$. For example, for weighted voting games a possible $v^{\prime}$ has been specified in Section 3.

Similarly, a splitting function on $\mathfrak{G}$,

$$
\sigma_{\mathfrak{G}}:\{\mathscr{G}=(N, v) \mid \mathscr{G} \in \mathfrak{G}\} \times N \times(\mathbb{N} \backslash\{0,1\}) \rightarrow \mathfrak{P}(\mathfrak{G}),
$$

turns a given coalitional game $\mathscr{G}=(N, v)$, a given player $i \in N$, and a given integer $m \geq 2$ into a set of new games of the form $\left(N^{\prime}, v^{\prime}\right)$, where player $i$ is split into $m$ players such that $N^{\prime}=\{n+$ $1, \ldots, n+m\} \cup(N \backslash\{i\})$ and $v^{\prime}: \mathfrak{P}\left(N^{\prime}\right) \rightarrow \mathbb{R}$ is the new coalitional function whose values are to be specified according to the type of games in class $\mathfrak{G}$. Again, for weighted voting games $v^{\prime}$ has been specified in Section 3, and for other classes of coalitional games, $v^{\prime}$ needs to be suitably defined. For example, if $\mathfrak{G}$ is the class of monotonic coalitional games, $v^{\prime}$ must be defined so that monotonicity is 
maintained, and since there are various possibilities of doing so, various distinct splitting functions can be defined for this class of games.

As an example, let $\mu_{\mathrm{wvg}}$ and $\sigma_{\mathrm{wvg}}$ denote the merging and splitting functions for weighted voting games as defined in Section 3. That is,

- for a weighted voting game $\mathscr{G}=\left(w_{1}, \ldots, w_{n} ; q\right)$ and a coalition $S \subseteq N=\{1, \ldots, n\}$, define $\mu_{\mathrm{wvg}}(\mathscr{G}, S)=\mathscr{G}_{\& S}$, and

- given a weighted voting game $\mathscr{G}=\left(w_{1}, \ldots, w_{n} ; q\right)$, a player $i$, and an integer $m \geq 2$, define $\sigma_{\mathrm{wvg}}(\mathscr{G}, i, m)$ to be the set of weighted voting games $\mathscr{G}_{i \div m}$.

We define the following properties of merging and splitting functions.

Definition 5.1. Let $\mathfrak{G}$ be a class of coalitional games and let $\mu_{\mathfrak{G}}$ be a merging function on $\mathfrak{G}$ and $\sigma_{\mathfrak{G}}$ be a splitting function on $\mathfrak{G}$.

1. We say $\mu_{\mathfrak{G}}$ satisfies consistency if for each $\mathscr{G}=(N, v) \in \mathfrak{G}$ and for each coalition $S \subseteq N$, if $\mu_{\mathfrak{G}}(\mathscr{G}, S)=\left(N^{\prime}, v^{\prime}\right)$ then $v(C \cup S)=v^{\prime}\left(C \cup\left\{i_{\& S}\right\}\right)$ holds for each coalition $C \subseteq N \backslash S$.

2. We say $\mu_{\mathfrak{G}}$ satisfies independence if for each $\mathscr{G}=(N, v) \in \mathfrak{G}$ and for each coalition $S \subseteq N$, if $\mu_{\mathfrak{G}}(\mathscr{G}, S)=\left(N^{\prime}, v^{\prime}\right)$ then $v(C)=v^{\prime}(C)$ holds for each coalition $C \subseteq N \backslash S$.

3. We say $\sigma_{\mathfrak{G}}$ satisfies consistency if for each $\mathscr{G}=(N, v) \in \mathfrak{G}$, for each player $i \in N$, and for each integer $m \geq 2$, if $\left(N^{\prime}, v^{\prime}\right) \in \sigma_{\mathfrak{G}}(\mathscr{G}, i, m)$ then $v(C \cup\{i\})=v^{\prime}(C \cup\{n+1, \ldots, n+m\})$ for each coalition $C \subseteq N \backslash\{i\}$.

4. We say $\sigma_{\mathfrak{G}}$ satisfies independence if for each $\mathscr{G}=(N, v) \in \mathfrak{G}$, for each player $i \in N$, and for each integer $m \geq 2$, if $\left(N^{\prime}, v^{\prime}\right) \in \sigma_{\mathfrak{G}}(\mathscr{G}, i, m)$ then $v(C)=v^{\prime}(C)$ for each coalition $C \subseteq N \backslash\{i\}$.

Intuitively, consistency means that the value of a coalition subject to merging or splitting should be the same before and after these operations. Independence means that the value of a coalition not affected by merging or splitting should remain the same in the new game, i.e., it depends only on the players in this coalition. In weighted voting games, both $\mu_{\mathrm{wvg}}$ and $\sigma_{\mathrm{wvg}}$ satisfy consistency and independence, since the weight of the new player in $\mu_{\mathfrak{G}}(\mathscr{G}, S)$ equals $\sum_{i \in S} w_{i}$ for merging, and since $\sum_{j=1}^{m} w_{n+j}=w_{i}$ for splitting.

The following example presents a merging function for the class of weighted majority games such that neither consistency nor independence is satisfied.

Example 5.2. Let $\mu_{\mathrm{wmg}}$ be the merging function that maps a given weighted majority game $\mathscr{G}=$ $\left(w_{1}, \ldots, w_{n}\right)$ and a given coalition $S \subseteq N$ to a new weighted majority game, where each player not in $S$ keeps his or her weight, and the new player $i_{\& S}$ merging $S$ receives weight $w_{i_{\& S}}=\prod_{i \in S} w_{i}$.

Consider the game $\mathscr{G}=(2,3,4,4)$ and the coalition $S=\{1,3\}$. Then, the game $\mu_{\mathrm{wmg}}(\mathscr{G}, S)=$ $(8,3,4)$ is formed. The value of the merged player in the new game is $v^{\prime}\left(\left\{i_{\& S}\right\}\right)=1$, whereas the value of $S$ in the original game is $v(S)=0$. Thus, $\mu_{\mathrm{wmg}}$ is not consistent. On the other hand, the value of the coalition of the other players $\left(\{2,4\}\right.$ in $\mathscr{G}$ and $\{2,3\}$ in $\left.\mu_{\mathrm{wmg}}(\mathscr{G}, S)\right)$ decreases from 1 to 0. Thus, $\mu_{\mathrm{wmg}}$ is not independent.

A similar example is obtained by using, e.g., the maximum or minimum weight of the coalition's players instead of the product of their weights, or any other function that is not additive. 
In general, a merging function $\mu_{\mathrm{csg}}$ on the class of constant-sum games (i.e., games $\mathscr{G}=(N, v)$ such that $v(C)+v(N \backslash C)=v(N)$ holds for each coalition $C \subseteq N)$ is neither consistent nor independent whenever for $\mathscr{G}=(N, v)$, some coalition $S \subseteq N$, and $\mu_{\mathrm{csg}}(\mathscr{G}, S)=\left(N^{\prime}, v^{\prime}\right)$, we have that $v(S) \neq v^{\prime}\left(\left\{i_{\& S}\right\}\right)$ and $v(N)=v^{\prime}\left(N^{\prime}\right)$, since then $v(N \backslash S) \neq v^{\prime}\left(N \backslash\left\{i_{\& S}\right\}\right)$.

As pointed out by an anonymous reviewer, natural merging and splitting functions may also exist in other important classes of coalitional games with transferable utilities where each player posesses a certain amount of a divisible resource, such as fractional matching games, bankruptcy games, or market games (see, e.g., Shoham \& Leyton-Brown, 2009). Moreover, one could consider the class of path-disruption games (Bachrach \& Porat, 2010; Rey \& Rothe, 2011, 2012; Marple, Rey, \& Rothe, 2014), where merging any two unconnected vertices might influence the value of a coalition of other players. An approach on how to define a merging and splitting function on network flow games can be found in Section 5.2.

\subsection{Beneficial Merging and Splitting Generalized: The Case of Two Players}

We can now define the beneficial merging and splitting problems in general. Let $\mu_{\mathfrak{G}}$ be a merging function and $\sigma_{\mathfrak{G}}$ be a splitting function on a class $\mathfrak{G}$ of coalitional games and let PI be a power index. Define the following generalized problems.

\begin{tabular}{|c|c|}
\hline \multicolumn{2}{|r|}{$\mu_{\mathfrak{G}}$-PI-BENEFICIALMERGE } \\
\hline Given: & A game $\mathscr{G}=(N, v)$ in $\mathfrak{G}$ and a nonempty coalition $S \subseteq N$ \\
\hline Question: & $\begin{array}{l}\text { Is it true that } \operatorname{PI}\left(\mu_{\mathfrak{G}}(\mathscr{G}, S), i_{\& S}\right)>\sum_{i \in S} \operatorname{PI}(\mathscr{G}, i) \text {, where } \mu_{\mathfrak{G}}(\mathscr{G}, S)=\left(N^{\prime}, v^{\prime}\right) \text { with } N^{\prime}= \\
\left\{i_{\& S}\right\} \cup(N \backslash S) \text { ? }\end{array}$ \\
\hline \multicolumn{2}{|r|}{$\sigma_{\mathfrak{G}}$-PI-BENEFICIALSPLIT } \\
\hline Given: & A game $\mathscr{G}=(N, v)$ in $\mathfrak{G}, N=\{1, \ldots, n\}$, a player $i \in N$, and an integer $m \geq 2$ \\
\hline Question: & $\begin{array}{l}\text { Is there a game } \mathscr{G}^{\prime}=\left(N^{\prime}, v^{\prime}\right) \in \sigma_{\mathfrak{G}}(\mathscr{G}, i, m) \text { with } N^{\prime}=\{n+1, \ldots, n+m\} \cup(N \backslash\{i\}) \\
\text { such that } \sum_{j=1}^{m} \operatorname{PI}\left(\mathscr{G}^{\prime}, n+j\right)>\operatorname{PI}(\mathscr{G}, i) \text { ? }\end{array}$ \\
\hline
\end{tabular}

Intuitively, $\mu_{\mathfrak{G}}$-PI-BENEFICIALMERGE is the problem of whether a coalition of players can benefit from merging via $\mu_{\mathfrak{G}}$ by raising their power in terms of $f$. Similarly, $\sigma_{\mathfrak{G}}$-PI-BENEFICIALSPLIT is the problem of whether a player can benefit from splitting into a number of new players via $\sigma_{\mathfrak{G}}$ by raising his or her power in terms of PI.

Generalizing Proposition 4.1, if consistency and independence are satisfied by the merging function, a coalition of two players cannot benefit from merging nor can a player benefit from splitting into two players considering the probabilistic Banzhaf index.

Theorem 5.3. Let $\mu_{\mathfrak{G}}$ be a merging function and let $\sigma_{\mathfrak{G}}$ be a splitting function, both satisfying consistency and independence.

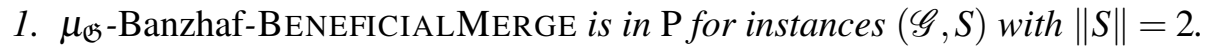

2. $\sigma_{\mathfrak{G}}$-Banzhaf-BeneficialSplit is in $\mathrm{P}$ for instances $(\mathscr{G}, i, 2)$.

Proof. Let $\mathscr{G}=(N, v)$ be a coalitional game and let $\mu_{\mathfrak{G}}$ be a consistent and independent merging function. Without loss of generality (see Footnote 8 ), let $S=\{n-1, n\}$. We obtain a new game 


$$
\begin{aligned}
& \mu_{\mathfrak{G}}(\mathscr{G}, S)=\left(\{1, \ldots, n-1\}, v^{\prime}\right) \text {, where } n-1 \text { is the new player merging } S \text { in } \mathscr{G} \text {. It holds that } \\
& \operatorname{Banzhaf}\left(\mu_{\mathfrak{G}}(\mathscr{G}, S), n-1\right)-(\operatorname{Banzhaf}(\mathscr{G}, n-1)+\operatorname{Banzhaf}(\mathscr{G}, n)) \\
& =\frac{1}{2^{n-1}}\left(\sum_{C \subseteq\{1, \ldots, n-2\}} 2\left(v^{\prime}(C \cup\{n-1\})-v^{\prime}(C)\right)\right. \\
& -\sum_{C \subseteq N \backslash\{n-1, n\}}(v(C \cup\{n-1\})-v(C))-\sum_{\substack{C \subseteq N \backslash\{n-1\}, n \in C}}(v(C \cup\{n-1\})-v(C)) \\
& \left.-\sum_{C \subseteq N \backslash\{n, n-1\}}(v(C \cup\{n\})-v(C))-\sum_{\substack{C \subseteq N \backslash\{n\} \\
n-1 \in C}}(v(C \cup\{n\})-v(C))\right) \\
& =\frac{1}{2^{n-1}}\left(\sum_{C \subseteq\{1, \ldots, n-2\}}(\underbrace{2 v^{\prime}(C \cup\{n-1\})-2 v(C \cup\{n-1, n\})}_{=0 \text { (by consistency) }}+\underbrace{2 v(C)-2 v^{\prime}(C)}_{=0 \text { (by independence) }})\right)=0 .
\end{aligned}
$$

In the case of splitting, consider a game $\mathscr{G}=(N, v)$ with $n$ players, a consistent and independent splitting function $\sigma_{\mathfrak{G}}$, and, without loss of generality, player $n$ in $\mathscr{G}$ splitting into players $n+1$ and $n+2$, which results in a new game $\mathscr{G}^{\prime} \in \sigma_{\mathfrak{G}}(\mathscr{G}, n, 2)$. Now, it similarly holds that

$$
\operatorname{Banzhaf}\left(\mathscr{G}^{\prime}, n+1\right)+\operatorname{Banzhaf}\left(\mathscr{G}^{\prime}, n+2\right)-\operatorname{Banzhaf}(\mathscr{G}, n)=0,
$$

as claimed.

In particular, this immediately implies Proposition 4.1 for $\mu_{\mathrm{wvg}}$ and $\sigma_{\mathrm{wvg}}$. As another example, we next consider threshold network flow games on hypergraphs, a class of compactly representable simple coalitional games.

\subsection{Example: Threshold Network Flow Games on Hypergraphs}

Bachrach and Rosenschein (2009) analyze threshold network flow games on graphs. A threshold network flow game (TNFG, for short) is defined on an edge-weighted graph with $n$ agents that each control one edge, a source vertex $s \in V$ and a target vertex $t \in V$, and a threshold $k \in \mathbb{R}$. The coalitional function is the success function, where a coalition of agents is successful if and only if a data flow of size $k$ can be sent from $s$ to $t$ over the edges represented by the agents in the coalition.

How can merging and splitting be defined in this setting? Since agents control single edges, ${ }^{11}$ merging two or more agents would yield one new agent who controls more than one edge and so would be qualitively different from the remaining agents. Similarly, splitting an agent into several subagents would mean to "split" the original agent's edge, and it is unclear how to do that. Our approach for solving this issue is to consider threshold network flow games on hypergraphs rather than on graphs. A hyperedge in a hypergraph is any subset of the vertex set (so a graph is the special case of a hypergraph with hyperedges of size two only). Of course, agents in a hypergraph can have control over hyperedges of different sizes, but that is merely a quantitative difference.

11. Kalai and Zemel (1982a, 1982b) propose a model where each agent controls a set of edges, not only a single edge. As suggested by an anonymous reviewer, a natural merging function would then assign a merging coalition to the union of sets of edges controlled by players in the coalition of the original game. 
Definition 5.4. A threshold hypergraph network flow game (THNFG, for short) $\mathscr{G}=(N, v)$ is set on a weighted hypergraph $H=(V, E)$ with vertex set $V$ and a set $E=\left\{e_{1}, \ldots, e_{n}\right\}$ of $n$ weighted hyperedges (where agent $i$ represents hyperedge $e_{i}$ ), a weight function $w: E \rightarrow \mathbb{N}$ (represented as a list $\left(w_{1}, \ldots, w_{n}\right)$ with $\left.w_{i}=w\left(e_{i}\right)\right)$, a source vertex $s \in V$ and a target vertex $t \in V$, and a threshold $k \in \mathbb{R}$. The coalitional function $v: \mathfrak{P}(N) \rightarrow\{0,1\}$ is defined by $v(C)=1$ if a data flow of size $k$ from $s$ to $t$ is possible in $H_{\mid C}$, the subhypergraph of $H$ induced by the hyperedge set $\left\{e_{i} \mid i \in C\right\}$, and $v(C)=0$ otherwise.

Example 5.5. The THNFG with four agents in Figure 1 may help to visualize the definitions and theorems given below. It shows the hypergraph (in the standard bipartite graph representation for hypergraphs) related to the game $\mathscr{G}=(\{1, \ldots, 5\}, v)$ with

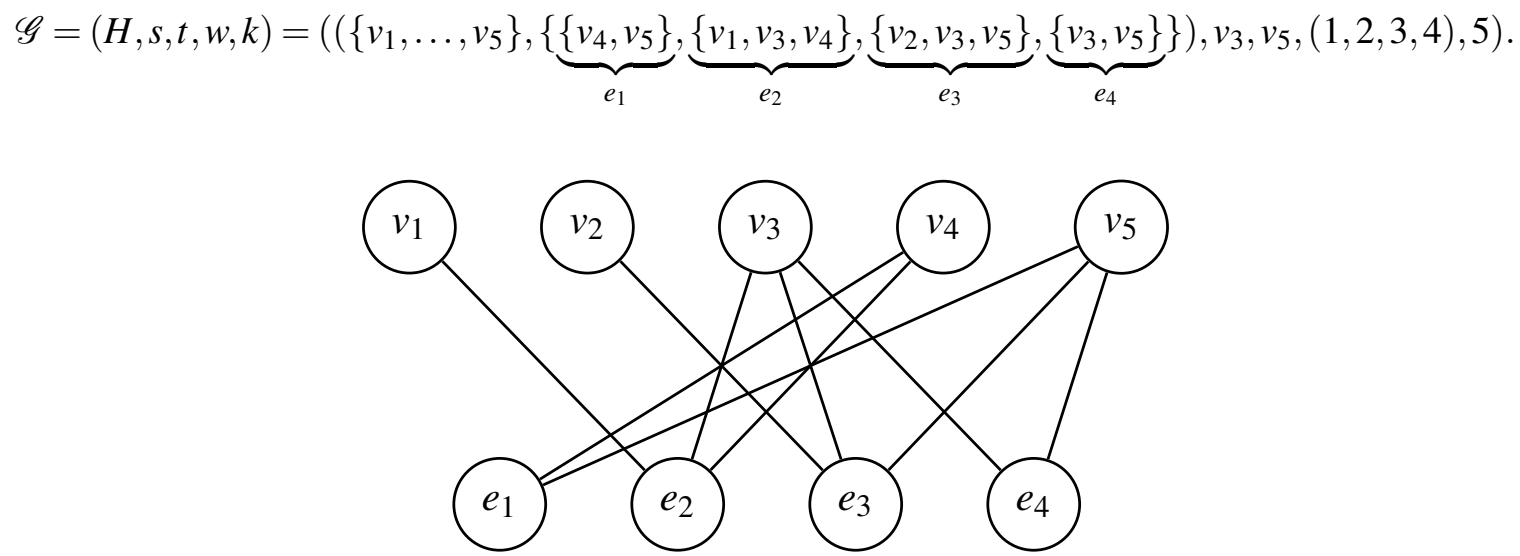

Figure 1: A THNFG for Example 5.5

Possible applications of THNFGs can be found in grid computing where many-often thousands of - computers collaborate to solve a common task by distributing various subtasks to certain clusters of computers, which represent the agents (respectively, the hyperedges). Connecting a number of such computer clusters corresponds to forming a coalition of agents. Modeled as a game, a coalition (i.e., a set of clusters) is successful if it connects the source to the target and allows a sufficient network data flow within the capacity of the computers in the clusters, and in this case these clusters can be assigned the desired subtask. As another example of a possible application of THNFGs, we propose to use this model for smart power grids that deliver electricity from suppliers to consumers. Success of a coalition would mean here that a certain threshold is to be exceeded according to the consumers' current demands, thus allowing a sufficiently large power flow.

For TNFGs, determining the raw Banzhaf index is \#P-complete, while the Shapley-Shubik index is only known to be at least as hard as problems in NP (Bachrach \& Rosenschein, 2009). However, for THNFGs, the following results are consequences of the corresponding results for weighted voting games. The easy hardness proof simply reduces the problem for WVGs to the corresponding problem for THNFGs by mapping a given WVG $\mathscr{G}=\left(w_{1}, \ldots, w_{n}, q\right)$ to the THNFG

$$
\mathscr{H}=\left(\left(\left\{v_{0}, v_{1}, \ldots, v_{n+1}\right\},\left\{e_{1}, \ldots e_{n}\right\}\right), v_{0}, v_{n+1},\left(w_{1}, \ldots, w_{n}\right), q\right),
$$

where $e_{i}=\left\{v_{0}, v_{i}, v_{n+1}\right\}$ for each $i, 1 \leq i \leq n$, with the same weights and threshold. Since the value of each coalition $C$ in $\mathscr{G}$ equals the value of $C$ in $\mathscr{H}$, we have $\operatorname{Banzhaf}^{*}(\mathscr{G}, i)=\operatorname{Banzhaf}^{*}(\mathscr{H}, i)$ 
and $\operatorname{ShapleyShubik}^{*}(\mathscr{G}, i)=\operatorname{ShapleyShubik}^{*}(\mathscr{H}, i)$ for each player $i$. Since this reduction is parsimonious, \#P-parsimonious-hardness of Banzhaf* for THNFGs is inherited from that for WVGs, and \#P-many-one-hardness of ShapleyShubik* for THNFGs is inherited from that for WVGs.

Proposition 5.6. Computing the raw Banzhaf power index in THNFGs is \#P-parsimonious-complete, while computing the raw Shapley-Shubik power index in THNFGs is \#P-many-one-complete.

The "power compare" problem for weighted voting games with respect to a power index PI (originally introduced by Faliszewski \& Hemaspaandra, 2009) can be defined analogously for THNFGs:

\section{PI-THNFG-POWERCOMPARE}

Given: $\quad$ Two THNFGs, $\mathscr{G}$ and $\mathscr{G}^{\prime}$, and a player $i$ occurring in both games.

Question: $\quad$ Is is true that $\operatorname{PI}(\mathscr{G}, i)>\operatorname{PI}\left(\mathscr{G}^{\prime}, i\right)$ ?

By suitably extending the reduction given right above Proposition 5.6, we have the following.

Corollary 5.7. PI-THNFG-POWERCOMPARE is PP-hard for PI $\in\{$ ShapleyShubik, Banzhaf $\}$.

The use of hyperedges makes it possible that, in THNFGs, a coalition of agents can be merged into a single new agent who controls the hyperedge that corresponds to the union of vertices belonging to the hyperedges of the coalition's original agents. In the example of grid computing, a merge of two clusters allows new connections as well as the sum of the computing power of the computers in the clusters as a total weight. Similarly, it is possible for an agent in such a setting to split into several subagents by partitioning this agent's hyperedge into subsets that each are controlled by one of the new subagents. We define the merging function and the splitting function for THNFGs as follows:

- The merging function $\mu_{\mathrm{thnfg}}$ on THNFGs maps a given THNFG $\mathscr{G}=(H, s, t, w, k)$, with hypergraph $H=(V, E)$, and a given coalition $S$ of agents to the new THNFG $\mu_{\text {thnfg }}(\mathscr{G}, S)=$ $\left(H_{\& S}, s, t, w_{\& S}, k\right)$, where the new hypergraph is $H_{\& S}=\left(V, E_{\& S}\right)$ with the new set of hyperedges $E_{\& S}=\left(E \backslash\left\{e_{i} \mid i \in S\right\}\right) \cup\left\{e_{\& S}\right\}$, the new agent $i_{\& S}$ controls hyperedge $e_{\& S}=\bigcup_{i \in S} e_{i}$, and the new weight function $w_{\& S}$ is given by $w_{\& S}\left(e_{i}\right)=w_{i}$ for $i \notin S$, and $w_{\& S}\left(e_{\& S}\right)=\sum_{i \in S} w_{i}$.

- The splitting function $\sigma_{\text {thnfg }}$ on THNFGs maps a given THNFG $\mathscr{G}=(H, s, t, w, k)$, with hypergraph $H=(V, E)$, a given agent $i$, and a given integer $m \geq 2$ to the new THNFG $\sigma_{\text {thnfg }}(\mathscr{G}, i, m)=\left(H_{i \div m}, s, t, w_{i \div m}, k\right)$, where the new hypergraph is $H_{i \div m}=\left(V, E_{i \div m}\right)$ with $E_{i \div m}=\left(E \backslash\left\{e_{i}\right\}\right) \cup\left\{e_{n+1}, \ldots, e_{n+m}\right\}$, agent $i$ is split into $m$ agents $n+1, \ldots, n+m$ such that $\bigcup_{j=1}^{m} e_{n+j}=e_{i}$ and $e_{n+j} \cap e_{\ell}=\emptyset$ for $\ell \in\{1, \ldots, n\} \backslash\{i\}$, and the new weight function $w_{i \div m}$ is given by $w_{i \div m}\left(e_{\ell}\right)=w_{\ell}$ for $\ell \neq i$, and the new agents' weights $w_{n+j}=w_{i \div m}\left(e_{n+j}\right)$, $1 \leq j \leq m$, satisfy $\sum_{j=1}^{m} w_{n+j}=w_{i}$.

In contrast to weighted voting games, consistency is not satisfied in general for THNFGs, neither by $\mu_{\text {thnfg }}$ nor by $\sigma_{\text {thnfg }}$. On the one hand, merging two agents via $\mu_{\text {thnfg }}$ can create new connections between vertices and thus allows new data flows to emerge. On the other hand, existing connections can get lost by splitting an agent via $\sigma_{\text {thnfg }}$ (i.e., splitting the corresponding hyperedge). Therefore, merging and splitting by $\mu_{\text {thnfg }}$ and $\sigma_{\text {thnfg }}$ might be advantageous for the probabilistic Banzhaf index, even for size-two coalitions or a split into two players. 
Example 5.8 (continuing Example 5.5). Consider again the THNFG $\mathscr{G}$ from Example 5.5. Merging the agents of coalition $S=\{1,3\}$ via $\mu_{\mathrm{thnfg}}$, allows new connections, but is disadvantagous for the probabilistic Banzhaf index in the new game

$$
\mu_{\mathrm{thnfg}}(\mathscr{G},\{1,3\})=\left(\left(\left\{v_{1} \ldots, v_{5}\right\},\{\underbrace{\left\{v_{2}, v_{3}, v_{4}, v_{5}\right\}}_{e_{\&\{1,3\}}}, \underbrace{\left\{v_{1}, v_{3}, v_{4}\right\}}_{e_{2}}, \underbrace{\left\{v_{3}, v_{5}\right\}}_{e_{4}}\}\right), v_{3}, v_{5},(4,2,4), 5\right) .
$$

However, merging the agents of coalition $S=\{1,2\}$ via $\mu_{\mathrm{thnfg}}$, is beneficial for the probabilistic Banzhaf index in the new game

$$
\mu_{\mathrm{thnfg}}(\mathscr{G},\{1,2\})=\left(\left(\left\{v_{1} \ldots, v_{5}\right\},\{\underbrace{\left\{v_{2}, v_{3}, v_{5}\right\}}_{e_{\&\{1,2\}}\left\{v_{1}, v_{3}, v_{4}, v_{5}\right\}}, \underbrace{\left\{v_{3}, v_{5}\right\}}_{e_{3}}\}\right), v_{1}, v_{5},(3,3,4), 5\right) .
$$

For comparison, let $\mathscr{G}^{\prime}$ be the corresponding weighted voting game with $\mathscr{G}^{\prime}=(1,2,3,4 ; 5)$ (i.e., with the same weights and threshold). Merging the agents of $S=\{1,3\}$ in $\mathscr{G}^{\prime}$ via $\mu_{\mathrm{wvg}}\left(\mathscr{G}^{\prime}, S\right)=\mathscr{G}_{\&\{1,3\}}^{\prime}=$ $(4,2,4 ; 5)$ as well as merging the agents of $S=\{1,2\}$ in $\mathscr{G}^{\prime}$ via $\mu_{\mathrm{wvg}}\left(\mathscr{G}^{\prime}, S\right)=\mathscr{G}_{\&\{1,2\}}^{\prime}=(3,3,4 ; 5)$ are neutral for the probabilistic Banzhaf index.

However, just as $\mu_{\mathrm{wvg}}$ and $\sigma_{\mathrm{wvg}}$ for weighted voting games, both $\mu_{\mathrm{thnfg}}$ and $\sigma_{\mathrm{thnfg}}$ satisfy independence for THNFGs: The value of a coalition only depends on the hyperedges of the agents within the coalition, not on other hyperedges that might have been merged or split.

\subsection{Merging and Splitting in Unanimity Games}

A simple game $\mathscr{G}=(N, v)$ is called a unanimity game if only the grand coalition wins, i.e., $v(C)=1$ if $C=N$, and $v(C)=0$ if $C \subsetneq N$. For example, a weighted voting game $\mathscr{G}=\left(w_{1}, \ldots, w_{n} ; q\right)$ is a unanimity weighted voting game if and only if $\sum_{i=1}^{n} w_{i}-\min _{i \in N} w_{i}<q \leq \sum_{i=1}^{n} w_{i}$.

There is only one possible merging function for unanimity games. Let $\mathscr{G}$ be a unanimity game and let $S \subseteq N$ be a coalition. Define $\mu_{\mathrm{ug}}(\mathscr{G}, S)=\left(N^{\prime}, v^{\prime}\right)$ by $N^{\prime}=\left\{i_{\& S}\right\} \cup(N \backslash S)$ and $v^{\prime}(C)=1$ if $C=N^{\prime}$, and $v^{\prime}(C)=0$ if $C \subsetneq N^{\prime}$. Obviously, $\mu_{\text {ug }}$ satisfies consistency and independence.

For unanimity weighted voting games, Aziz et al. (2011) show that for the normalized Banzhaf index, merging is always disadvantageous, whereas splitting is always advantageous. (Thus one can decide in polynomial time whether or not merging or splitting is beneficial.) In strong contrast, we show that in unanimity games with respect to the probabilistic Banzhaf index, splitting is always disadvantageous or neutral, whereas merging is neutral for size-two coalitions, yet advantageous for coalitions with at least three players.

Theorem 5.9. Let $\mathscr{G}$ be a unanimity game with player set $N$.

1. $(\mathscr{G}, S) \notin \mu_{\mathrm{ug}}$-Banzhaf-BenEFICIALMERGE for each $S \subseteq N$ with $\|S\|=2$,

2. $(\mathscr{G}, S) \in \mu_{\mathrm{ug}}$-Banzhaf-BeneficialMerGe for each $S \subseteq N$ with $\|S\| \geq 3$.

3. $(\mathscr{G}, i, m) \notin \sigma_{\mathrm{ug}}$-Banzhaf-BENEFICIALSPLIT for each $i \in N$ and $m \geq 2$.

Proof. The first statement follows immediately from Theorem 5.3.

To prove the second statement, note that in a unanimity game, any player $i$ can be pivotal only for the coalition $S=N \backslash\{i\}$, and $i$ always is pivotal for this coalition. Thus the raw Banzhaf 
index of each $i$ is always equal to one. It follows that $\operatorname{Banzhaf}(\mathscr{G}, i)=1 / 2^{n-1}$ for each player $i \in N$. If an arbitrary coalition $S$ merges, the Banzhaf index of a player $i$ in the new game $\mu_{\mathrm{ug}}(\mathscr{G}, S)$ is $\operatorname{Banzhaf}\left(\mu_{\mathrm{ug}}(\mathscr{G}, S), i\right)=1 / 2^{n-\|S\|}$. Since $\|S\| \geq 3$, we have

$$
\operatorname{Banzhaf}\left(\mu_{\mathrm{ug}}(\mathscr{G}, S), i_{\& S}\right)-\sum_{i \in S} \operatorname{Banzhaf}(\mathscr{G}, i)=\frac{2^{\|S\|-1}-\|S\|}{2^{n-1}}>0 .
$$

The third statement can be shown by similar arguments. In particular, for any possible split into players with integer weights, we have

$$
\sum_{j=1}^{m} \operatorname{Banzhaf}\left(\sigma_{\text {ug }}(\mathscr{G}, i, m), n+j\right)-\operatorname{Banzhaf}(\mathscr{G}, i)=\frac{m-2^{m-1}}{2^{n+m-2}} \leq 0 .
$$

This completes the proof.

\section{Conclusions and Future Work}

We have analyzed the beneficial merging, splitting, and annexation problems in terms of their complexity. In particular, our results complement-by considering the probabilistic Banzhaf power index - those by Aziz et al. (2011) for the normalized Banzhaf power index in weighted voting games. To some extent, these results differ from those for the normalized Banzhaf power index: For the probabilistic Banzhaf power index, beneficial merging and splitting turns out to be tractable for a merger of size-two coalitions and a split into two players.

One of our main results is that, solving previous conjectures in the affirmative, we have pinpointed the precise complexity of the beneficial merging problem in weighted voting games for the Shapley-Shubik and the probabilistic Banzhaf index by showing that it is PP-complete in both cases. On the one hand, this result is interesting in itself from a theoretical point of view. On the other hand, this provides a PP-completeness result for a natural problem from game theory, which are by far rarer than NP-complete problems in this field. Since merging can be seen as manipulative behavior, a high complexity can be interpreted as a protection shield against such strategic interference. While there are several known methods to circumvent NP-hardness-such as approximation, fixedparameter tractability, typical case analyses (for a discussion of applying such methods to NP-hard voting problems see, e.g., Rothe \& Schend, 2013), or a recent algebraic approach (Berghammer \& Schnoor, 2014)—, such methods are less applicable to circumvent hardness for higher complexity classes. Since PP is considered to be a much larger complexity class than NP, PP-hardness can be seen as leading to a potentially higher degree of protection than mere NP-hardness. Still, the hardness of our problems rests on the hardness to compute the related power indices. Note that there are good approximation schemes and dynamic methods known for computing the Shapley-Shubik index (see, e.g., Bachrach, Markakis, Resnik, Procaccia, Rosenschein, \& Saberi, 2010; Fatima, Wooldridge, \& Jennings, 2008; Bilbao, Fernández, Jiménez, \& López, 2000; Matsui \& Matsui, 2000; Shapley, 1953), though there is not much known about the exact case.

We have obtained the same PP-completeness result for beneficial splitting (a.k.a. false-name manipulation) whenever the new players' weights are given. For a given number of false identities, but unknown weights, we raised the lower bound (known for the Shapley-Shubik index) from NPhardness to PP-hardness and showed that it is contained in $\mathrm{NP}^{\mathrm{PP}}$ whenever the number of false 
identities is given in unary. For this problem, it remains open whether it can be shown to be complete for $\mathrm{NP}^{\mathrm{PP}}$, a huge complexity class that—by Toda's theorem (1991)—contains the entire polynomial hierarchy. $\mathrm{NP}^{\mathrm{PP}}$ is an interesting class, but somewhat sparse in natural complete problems. The only (natural) $\mathrm{NP}^{\mathrm{PP}}$-completeness results we are aware of are due to Littman et al. (1998), who analyze a variant of the satisfiability problem and questions related to probabilistic planning, and due to Mundhenk et al. (2000), who study problems related to finite-horizon Markov decision processes.

Another interesting open question is whether our results can be transferred also to the beneficial merging and splitting problems for the normalized Banzhaf index or other power indices.

For the beneficial annexation problem with a takeover of a single player, we showed NPcompleteness for both the Shapley-Shubik and the probabilistic Banzhaf index.

Finally, we have proposed a general framework for merging and splitting that can be applied to various classes of coalitional games with transferable utilities. An interesting task for future research is to study useful properties of merging and splitting functions, such as consistency and independence, in general and when applied to particular classes of games like network flow games or market games. Another interesting question, raised by an anonymous reviewer, is how to naturally extend the idea of merging to classes of games where players control several resources. Which properties do we want to hold in that case? Also, can a merging function that satisfies independence and consistence be unique for a certain class of games? For unanimity games we have observed that there is only one possible merging function that guarantees unanimity. For weighted voting games, however, such a uniqueness result does not hold, since there are different ways to distribute the player's weights that lead to the same coalitional function. For instance, the games $(1,3,4 ; 8)$ and $(2,3,4 ; 8)$ are semantically the same, even if players merge. Restricting to other classes or requiring other properties might imply uniqueness. Although consistency seems to be an essential property for a merging or splitting function, we have seen a natural merging function on threshold hypergraph network flow games that does not satisfy this property, and we made similar observations for other classes of games.

\section{Acknowledgments}

Preliminary versions of parts of this paper appear in the proceedings of the 19th European Conference on Artificial Intelligence (ECAI'10) (Rey \& Rothe, 2010a), the 5th European Starting AI Researcher Symposium (STAIRS'10) (Rey \& Rothe, 2010b), and the 11th Latin American Theoretical Informatics Symposium (LATIN'14) (Rey \& Rothe, 2014). We are grateful to the anonymous JAIR, ECAI'10, STAIRS'10, LATIN'14, and CoopMAS'14 reviewers for their helpful comments on this paper. This work was supported in part by DFG grants RO 1202/11-1, RO 1202/12-1 (within the ESF EUROCORES program LogICCC), and RO 1202/14-1.

\section{References}

Aziz, H., Bachrach, Y., Elkind, E., \& Paterson, M. (2011). False-name manipulations in weighted voting games. Journal of Artificial Intelligence Research, 40, 57-93.

Aziz, H., Brandt, F., \& Brill, M. (2013). The computational complexity of random serial dictatorships. Economic Letters, 121(3), 341-345. 
Aziz, H., \& Paterson, M. (2009). False name manipulations in weighted voting games: Splitting, merging and annexation. In Proceedings of the 8th International Joint Conference on Autonomous Agents and Multiagent Systems, pp. 409-416. IFAAMAS.

Bachrach, Y., \& Elkind, E. (2008). Divide and conquer: False-Name manipulations in weighted voting games. In Proceedings of the 7th International Joint Conference on Autonomous Agents and Multiagent Systems, pp. 975-982. IFAAMAS.

Bachrach, Y., Elkind, E., Meir, R., Pasechnik, D., Zuckerman, M., Rothe, J., \& Rosenschein, J. (2009). The cost of stability in coalitional games. In Proceedings of the 2nd International Symposium on Algorithmic Game Theory, pp. 122-134. Springer-Verlag Lecture Notes in Computer Science \#5814.

Bachrach, Y., Markakis, E., Resnik, E., Procaccia, A., Rosenschein, J., \& Saberi, A. (2010). Approximating power indices: Theoretical and empirical analysis. Journal of Autonomous Agents and Multi-Agent Systems, 20(2), 105-122.

Bachrach, Y., \& Porat, E. (2010). Path disruption games. In Proceedings of the 9th International Joint Conference on Autonomous Agents and Multiagent Systems, pp. 1123-1130. IFAAMAS.

Bachrach, Y., \& Rosenschein, J. (2009). Power in threshold network flow games. Journal of Autonomous Agents and Multi-Agent Systems, 18(1), 106-132.

Banzhaf III, J. (1965). Weighted voting doesn't work: A mathematical analysis. Rutgers Law Review, 19, 317-343.

Berghammer, R., \& Schnoor, H. (2014). Control of condorcet voting: Complexity and a relationalgebraic approach (extended abstract). In Proceedings of the 13th International Joint Conference on Autonomous Agents and Multiagent Systems, pp. 1365-1366. IFAAMAS.

Bilbao, J., Fernández, J., Jiménez, N., \& López, J. (2000). Generating functions for computing power indices efficiently. Top, 8(2), 191-213.

Bilbao, J., Fernández, J., Jiménez, N., \& López, J. (2002). Voting power in the European Union enlargement. European Journal of Operational Research, 143(1), 181-196.

Chalkiadakis, G., Elkind, E., \& Wooldridge, M. (2011). Computational Aspects of Cooperative Game Theory. Synthesis Lectures on Artificial Intelligence and Machine Learning. Morgan and Claypool Publishers.

Deng, X., \& Papadimitriou, C. (1994). On the complexity of comparative solution concepts. Mathematics of Operations Research, 4(2), 257-266.

Dubey, P., \& Shapley, L. (1979). Mathematical properties of the Banzhaf power index. Mathematics of Operations Research, 4(2), 99-131.

Elkind, E., Chalkiadakis, G., \& Jennings, N. (2008). Coalition structures in weighted voting games. In Proceedings of the 18th European Conference on Artificial Intelligence, pp. 393-397. IOS Press.

Elkind, E., Goldberg, L., Goldberg, P., \& Wooldridge, M. (2009). On the computational complexity of weighted voting games. Annals of Mathematics and Artificial Intelligence, 56(2), 109-131. 
Elkind, E., Pasechnik, D., \& Zick, Y. (2013). Dynamic weighted voting games. In Proceedings of the 12th International Joint Conference on Autonomous Agents and Multiagent Systems, pp. 515-522. IFAAMAS.

Faliszewski, P., \& Hemaspaandra, L. (2009). The complexity of power-index comparison. Theoretical Computer Science, 410(1), 101-107.

Fatima, S., Wooldridge, M., \& Jennings, N. (2008). A linear approximation method for the shapley value. Artificial Intelligence, 172(14), 1673-1699.

Felsenthal, D., \& Machover, M. (1995). Postulates and paradoxes of relative voting power - A critical re-appraisal. Theory and Decision, 38(2), 195-229.

Felsenthal, D., \& Machover, M. (2005). Voting power measurement: A story of misreinvention. Social Choice and Welfare, 25(2), 485-506.

Garey, M., \& Johnson, D. (1979). Computers and Intractability: A Guide to the Theory of NPCompleteness. W. H. Freeman and Company.

Gill, J. (1977). Computational complexity of probabilistic Turing machines. SIAM Journal on Computing, 6(4), 675-695.

Hunt, H., Marathe, M., Radhakrishnan, V., \& Stearns, R. (1998). The complexity of counting problems. SIAM Journal on Computing, 27(4), 1142-1167.

Kalai, E., \& Zemel, E. (1982a). Generalized network problems yielding totally balanced games. Operations Research, 30(5), 998-1008.

Kalai, E., \& Zemel, E. (1982b). Totally balanced games and games of flow. Mathematics of Operations Research, 7(3), 476-478.

Karp, R. (1972). Reducibility among combinatorial problems. In Miller, R., \& Thatcher, J. (Eds.), Complexity of Computer Computations, pp. 85-103. Plenum Press.

Littman, M., Goldsmith, J., \& Mundhenk, M. (1998). The computational complexity of probabilistic planning. Journal of Artificial Intelligence Research, 9(1), 1-36.

Marple, A., Rey, A., \& Rothe, J. (2014). Bribery in multiple-adversary path-disruption games is hard for the second level of the polynomial hierarchy (extended abstract). In Proceedings of the 13th International Joint Conference on Autonomous Agents and Multiagent Systems. IFAAMAS. To appear.

Matsui, T., \& Matsui, Y. (2000). A survey of algorithms for calculating power indices of weighted majority games. Journal of the Operation Research Society of Japan, 43(1), 71-86.

Mundhenk, M., Goldsmith, J., Lusena, C., \& Allender, E. (2000). Complexity results for finitehorizon Markov decision process problems. Journal of the ACM, 47(4), 681-720.

Nisan, N., Roughgarden, T., Tardos, É., \& Vazirani, V. (Eds.). (2007). Algorithmic Game Theory. Cambridge University Press.

Papadimitriou, C. (1995). Computational Complexity (Second edition). Addison-Wesley.

Peleg, B., \& Sudhölter, P. (2003). Introduction to the Theory of Cooperative Games. Kluwer Academic Publishers.

Penrose, L. (1946). The elementary statistics of majority voting. Journal of the Royal Statistical Society, 109(1), 53-57. 
Prasad, K., \& Kelly, J. (1990). NP-completeness of some problems concerning voting games. International Journal of Game Theory, 19(1), 1-9.

Rey, A., \& Rothe, J. (2010a). Complexity of merging and splitting for the probabilistic Banzhaf power index in weighted voting games. In Proceedings of the 19th European Conference on Artificial Intelligence, pp. 1021-1022. IOS Press.

Rey, A., \& Rothe, J. (2010b). Merging and splitting for power indices in weighted voting games and network flow games on hypergraphs. In Proceedings of the 5th European Starting AI Researcher Symposium, pp. 277-289. IOS Press.

Rey, A., \& Rothe, J. (2011). Bribery in path-disruption games. In Proceedings of the 2nd International Conference on Algorithmic Decision Theory, pp. 247-261. Springer-Verlag Lecture Notes in Artificial Intelligence \#6992.

Rey, A., \& Rothe, J. (2012). Probabilistic path-disruption games. In Proceedings of the 20th European Conference on Artificial Intelligence, pp. 923-924. IOS Press. An extended version appears in the proceedings of the 6th European Starting AI Researcher Symposium, IOS Press, pages 264-269, August 2012.

Rey, A., \& Rothe, J. (2014). False-name manipulation in weighted voting games is hard for probabilistic polynomial time. In Proceedings of the 11th Latin American Theoretical Informatics Symposium, pp. 60-71. Springer-Verlag Lecture Notes in Computer Science \#8392.

Rothe, J. (2005). Complexity Theory and Cryptology. An Introduction to Cryptocomplexity. EATCS Texts in Theoretical Computer Science. Springer-Verlag.

Rothe, J., \& Schend, L. (2013). Challenges to complexity shields that are supposed to protect elections against manipulation and control: A survey. Annals of Mathematics and Artificial Intelligence, 68(1-3), 161-193.

Shapley, L. (1953). A value for $n$-person games. In Kuhn, H., \& Tucker, A. (Eds.), Contributions to the Theory of Games, Vol. II of Annals of Mathematics Studies 40. Princeton University Press.

Shapley, L., \& Shubik, M. (1954). A method of evaluating the distribution of power in a committee system. The American Political Science Review, 48(3), 787-792.

Shoham, Y., \& Leyton-Brown, K. (2009). Multiagent Systems. Algorithmic, Game-Theoretic, and Logical Foundations. Cambridge University Press.

Toda, S. (1991). PP is as hard as the polynomial-time hierarchy. SIAM Journal on Computing, 20(5), 865-877.

Valiant, L. (1979). The complexity of computing the permanent. Theoretical Computer Science, 8(2), 189-201.

Wagner, K. (1986). The complexity of combinatorial problems with succinct input representations. Acta Informatica, 23, 325-356.

Zankó, V. (1991). \#P-completeness via many-one reductions. International Journal of Foundations of Computer Science, 2(1), 76-82.

Zuckerman, M., Faliszewski, P., Bachrach, Y., \& Elkind, E. (2012). Manipulating the quota in weighted voting games. Artificial Intelligence, 180-181(0), 1-19. 